\title{
Die Tiefenwasserableitung, ein Weg zur Sanierung von Seen
}

\author{
VON RENE GÄCHTER \\ Eidg. Anstalt für Wasserversorgung, Abwasserreinigung und Gewässerschutz (EAWAG) \\ an der ETH, 8600 Dübendorf \\ Manuskript eingegangen am 30.Januar 1976
}

\section{ABSTRACT \\ Lake Restoration by Bottom Water Siphoning}

This study shows that it is possible to restore a highly eutrophic lake by the mean of bottom water siphoning. The main effects of the hypolimnion drainage, such as, erosion of the hypolimnion and increase of the nutrient export out of the lake are discussed.

From mass balance calculations it is deduced that in eutrophic shallow lakes the nutrient supply to the trophogenic layer by the sediments can exceed temporarily the external loading by more than a hundredfold. The process of a lakes selfmaintaining auteutrophication is described. An extended summary in English is given at the end of the paper.

\section{Einleitung}

Der Mauensee liegt 2,5 km westlich Sursee, eingebettet in einem hauptsächlich landwirtschaftlich genutzten, muldenförmigen Einzugsgebiet von $4,3 \mathrm{~km}^{2}$ Ausdehnung (Abb.1). Seine Oberfläche beträgt $0,51 \mathrm{~km}^{2}$, die mittlere Tiefe $3 ; 9 \mathrm{~m}$, die

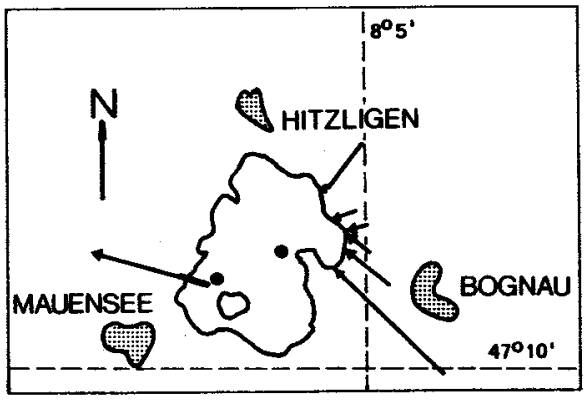

Abb. 1. Geographische Lage des Sees und der Meßstellen.

Fig. 1. Location of the lake and the sampling stations. 
maximale Tiefe 6,8 $\mathrm{m}$ und die mittlere Aufenthaltszeit des Wassers (Volumen/Abfluss) etwa 0,6 Jahre. Die Nährstoffzufuhr liegt im Bereich von $0,7 \mathrm{~g} \mathrm{P} / \mathrm{m}^{2} \mathrm{Jahr}$ und $19 \mathrm{~g} \mathrm{~N} / \mathrm{m}^{2} \mathrm{Jahr}[5,6]$. In den Jahren $1967 \mathrm{bis} 1969$ wurde das Phytoplankton weitgehend durch die Burgunderblutalge Oscillatoria rubescens dominiert [23]. Häufige Wasserblüten, die den See rot färbten und die Transparenz bis auf $30 \mathrm{~cm}$ reduzierten, prägten den optischen Charakter dieses hoch eutrophen Sees. Während der Sommerstagnation war sein Hypolimnion während mehrerer Monate anaerob, was die Rücklösung von Nährstoffen aus den Sedimenten begünstigte und sich bei der geringen Tiefe des Sees direkt produktionsfördernd auswirkte.

Der Nährstoffgehalt eines Sees ist abhängig von der externen Nährstoffzufuhr, der Nährstoffbelastung durch die Sedimente, der Nährstoffsedimentation und dem Nährstoffexport aus dem See via Ausfluss. Während der Stagnationsperiode führen Sedimentations-, Mineralisations- und Rücklösungsprozesse zu einer Nährstoffakkumulation im Hypolimnion. Die Ableitung von hypolimnischem Wasser anstelle von nährstoffärmerem Oberflächenwasser ist daher gleichbedeutend mit einem erhöhten Nährstoffexport aus dem See. Es war das Ziel dieser Untersuchung, in Anlehnung an die Arbeiten von Olszewski [13, 14], Eschmann [4] und PeCHLANER [15] am Beispiel des Mauensees zu untersuchen, ob und wieweit sich der trophische Charakter eines Sees durch diese Massnahme allein verändern lässt.

$\mathrm{Zu}$ diesem $Z_{\text {weck }}$ wurde von der tiefsten Stelle des Sees durch das Abflusswehr ein Rohr von $30 \mathrm{~cm}$ Durchmesser verlegt, durch welches, infolge eines statischen Uberdrucks von etwa $0,5 \mathrm{~m}$ Wassersäule, $4 \mathrm{~m}^{3}$ Tiefenwasser pro Minute in den Ausfluss gelangen (siehe Abb. 2).

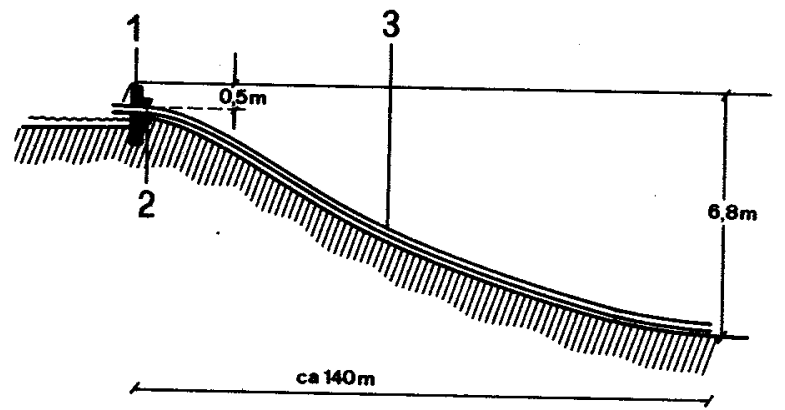

Abb. 2. Schematisches Längenprofil der Ablaufleitung: $I$ Mauer, 2 Schieber, 3 Leitung.

Fig. 2. Diagram of the siphon: $l$ dam, 2 valve, 3 pipe:

Die Leitung wurde im April 1968 in Betrieb genommen. In den Jahren 1968, 1969, 1970, 1971 und 1974 wurden in der eisfreien Periode in etwa monatlichen Abständen Proben erhoben. 1975 wurde Ende August eine einmalige Probenahme durchgeführt. Es wurden dabei die folgenden Komponenten regelmässig erfasst: Temperatur [1], Sichttiefe, Sauerstoff [22], Alkalität [17], pH, Nitrat [11], Nitrit [20], Kjeldahl-N [18], Phosphat [2], Gesamtphosphor im Filtrat und im Rohwasser [16]. Zusätzlich wurde in den Jahren 1971 und 1974 die Primärproduktion [19] gemessen. Die Tagesproduktionsraten wurden nach der von GÄCHTER [8] in Abb. 11 vorgeschlagenen Methode aus vierstündigen In-situ-Experimenten ermittelt.
Nachdem sich aus einer einjährigen Paralleluntersuchung von zwei Meßstellen keine gesicherten Unterschiede zwischen den beiden Messpunkten ergaben, wurde ab 1969 nur noch die dem Auslauf näherliegende Stelle untersucht (siehe Abb. 1).

\section{Resultate}

Bezüglich der Temperatur oberflächennaher Schichten unterscheiden sich die verschieden beobachteten Jahre dadurch, dass sich das Wasser 1968 und 1969 rascher und stärker erwärmte als in den übrigen Jahren (Abb.3). Die gemessenen Maximaltemperaturen betrugen etwa $26^{\circ}(1968), 26^{\circ}(1969), 24^{\circ}(1970), 23^{\circ}(1971)$ und $22^{\circ}(1974)$. Anderseits wurden in den Jahren 1971 und 1974 in den tieferen Wasserschichten deutlich höhere Temperaturen gemessen als in den vorangegangenen Jahren. Aus der flacheren Temperaturverteilung in den Sommern 1971 und 1974 kann gegenüber den Vorjahren ein erhöhter Austausch zwischen boden- und oberflächennahen Wassermassen abgeleitet werden. Aufgrund der Temperaturverteilung muss angenommen werden, dass die Sommerstagnation 1969 und 1974 bereits in der zweiten Hälfte März, 1970 und 1971 erst etwa Mitte April einsetzte. Herbstvollzirkulation wurde in den Jahren 1968 bis 1970 etwa Mitte Oktober, 1971 und 1974 bereits Anfang Oktober erreicht. Der Beginn der Herbstteilzirkulation (Abkühlung an der Oberfläche) fält in den Jahren 1968 bis 1971 in die Periode Mitte Juli bis Anfang August. 1974 erwärmte sich das Wasser nach einer ersten Abkühlung im Juli nochmals und erreichte maximale Werte erst in der zweiten Hälfte des Monats August.

Daraus folgt, dass zwischen dem Beginn der Sommerstagnation und dem Einsetzen der Teilzirkulation etwa die folgenden Zeitspannen liegen:

\begin{tabular}{lllll}
1968 & 1969 & 1970 & 1971 & 1974 \\
\hline 3 Monate & 4 Monate & 3,5 Monate & 2,5 Monate & $\begin{array}{l}3,5 \text { Monate } \\
\text { (5 Monate) }\end{array}$
\end{tabular}

Die gemessenen Sauerstoffkonzentrationen sind in Abb. 3 graphisch zusammengestellt. Maximale Sauerstoffkonzentrationen wurden in oberflächennahen Schichten alljährlich in der Periode April bis Juni beobachtet. Während in den Jahren 1968 bis 1970 Werte von mehr als $20 \mathrm{mg} \mathrm{O} / 1$ beobachtet wurden, wurden 1971 $15 \mathrm{mg} \mathrm{O} \mathrm{O}_{2} / 1$ nicht erreicht und 1974 nur knapp überschritten. Die beobachteten Maximalwerte betrugen $196826,7 \mathrm{mg} \mathrm{O} / 1,196924,3 \mathrm{mg} \mathrm{O} / 1,197022 \mathrm{mg} \mathrm{O} / \mathrm{l}$, $197113,8 \mathrm{mg} \mathrm{O}_{2} / 1$ und $197416,8 \mathrm{mg} \mathrm{O} / 1$. Allerdings gilt es zu berücksichtigen, dass mit der nur monatlichen Entnahme von Stichproben nicht die ganze Breite der aufgetretenen Schwankungen im Epilimnion erfasst wurde.

Parallel zur Abnahme dieser Sauerstoffmaxima im Epilimnion ist das Sauerstoffdefizit im Hypolimnion kleiner geworden. Das Sauerstoffdefizit im Hypolimnion ist das über die Dauer der Stagnationsperiode integrierte Resultat aller sauerstoffzehrenden Prozesse. Da sich die Sauerstoffkonzentration des Hypolimnions mit zunehmender Dauer der Stagnationsperiode stetig verringert, sind die in den tieferen Wasserschichten gewonnenen Sauerstoffresultate weniger von Zufälligkeiten der Probenahme abhängig. Im Jahre 1968 lagen die Sauerstoffkonzentrationen in 

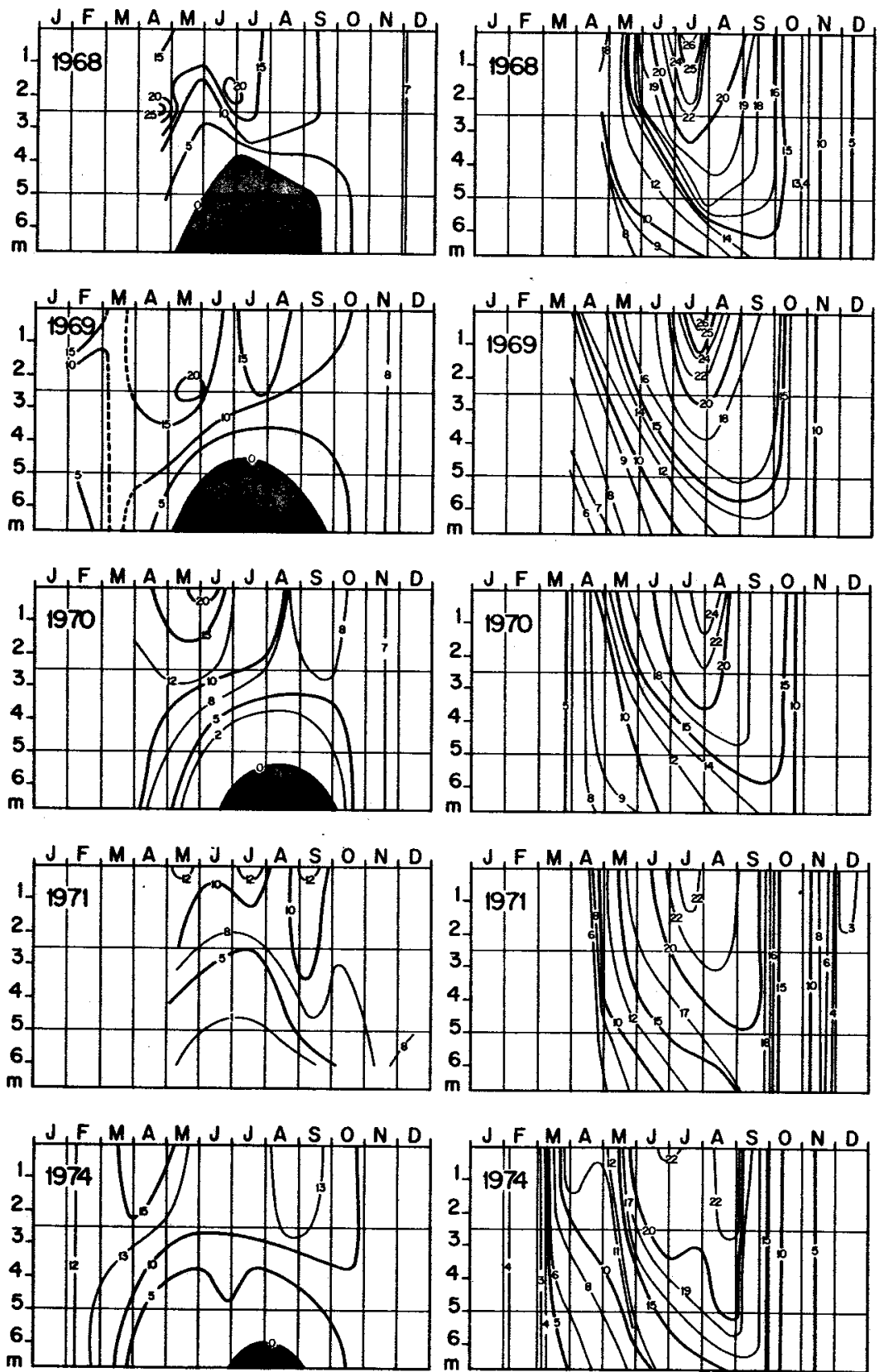

Abb. 3. Variation der Sauerstoffkonzentration (links) und der Temperatur (rechts) in Abhängigkei von Jahreszeit und Seetiefe. Angaben und $\mathrm{mg} \mathrm{O}_{2} / 1$ bzw. ${ }^{\circ} \mathrm{C}$.

Fig. 3. Seasonal variation of temperature (right) and oxygen concentration (left). Figures indicate ${ }^{\circ} \mathrm{C}$ and $\mathrm{mg} \mathrm{O}_{2} / \mathrm{l}$ respectively.
$5 \mathrm{~m}$ Tiefe während 3 Monaten, im Jahre 1969 noch etwa während 2 Monaten unter der Nachweisbarkeitsgrenze. Seit 1970 konnte in dieser Tiefe während des ganzen Jahres immer Sauerstoff nachgewiesen werden. Die minimalen Werte lagen 1970 bei $0,17 \mathrm{mg} \mathrm{O}_{2} / 1,19710,3 \mathrm{mg} \mathrm{O} / 1$ und 1974 bei $0,8 \mathrm{mg} \mathrm{O}_{2} / 1$. Dass 1971 überhaupt keine anaerobe Zone beobachtet wurde, mag damit zusammenhängen, dass in diesem Jahr die "Grundproben» in etwa $6 \mathrm{~m}$ statt knapp über Grund in $6,5 \mathrm{~m}$ entnommen wurden. 1975 wurden Ende Juli in $5 \mathrm{~m}$ Tiefe noch $3,0 \mathrm{mg} \mathrm{O}_{2} / 1$, in $6,8 \mathrm{~m}$ Tiefe immer noch $1,1 \mathrm{mg} \mathrm{O} / 1$ gemessen. Die Resultate zeigen deutlich, dass die Tiefe immer noch $1,1 \mathrm{mg} \mathrm{O}_{2} / 1$ gemessen. Die Resultate zeigen deutlich, dass die anaerobe Zon Die jahreszeitlichen Veränderungen der Sichttiefen (Abb.4) in den Jahren
$967 / 68$ sind charakterisiert durch kurzfristige Schwankungen mit hohen Ampli-

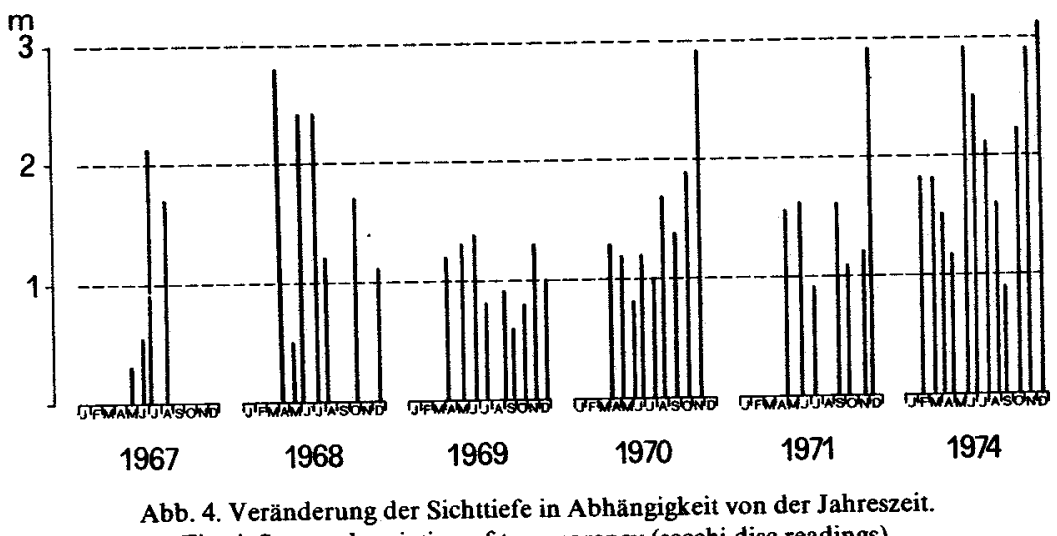

Fig. 4. Seasonat variauion of transparency (secchi disc readings).

tuden. Kleine Sichttiefen fielen stets mit hohen Phytoplanktondichten zusammen. Die Jahre 1969-1971 verhielten sich diesbezüglich wesentlich ausgeglichener. Im Jahre 1974 wurde im Juni wieder ein deutliches Sommermaximum beobachtet. Die im Verlaufe eines Jahres beobachtete minimale Sichttiefe ist insofern aufschlussreich, als sie indirekt Aufschluss gibt über die unter optimalen Bedingungen.mögliche maximale Planktondichte. Während der Beobachtungsperiode wurden die folgenden Sichttiefenminima beobachtet: $19670,3 \mathrm{~m} ; 19680,4 \mathrm{~m} ; 19690,6 \mathrm{~m} ; 1970$ $0,8 \mathrm{~m} ; 19710,9 \mathrm{~m} ; 19740,9 \mathrm{~m}$. Während im Beobachtungsjahre 1968 dreimal, 1970 und 1971 nur je einmal Sichttiefen von mehr als zwei Metern beobachtet wurden, wurde 1974 dieser Wert sechsmal überschritten. Die maximal beobachteten Werte betrugen $19682,8 \mathrm{~m}, 19691,4 \mathrm{~m}, 1970$ und 1971 je 2,9 $\mathrm{m}$ und 1974 3,1 m.

Die jahreszeitlichen Veränderungen der Gesamtphosphorkonzentration sind aus Abb 5 ersichtlich. Da diese Komponente 1969 nur lückenhaft bestimmt wurde, wurde für dieses Jahr auf eine graphische Darstellung der Resultate verzichtet. Die Jahre 1968 und 1970 zeichnen sich gegenüber den folgenden Jahren im Zeitabschnitt Juli/August durch einen vom Sediment ausgehenden und die gesamte Wassermasse erfassenden Konzentrationsanstieg aus. In diesem Zeitabschnitt erreichte die Gesamtphosphorkonzentration im Jahre 1968 in $2,5 \mathrm{~m}$ Tiefe $2280 \mu \mathrm{g}$ P/1, 1970 


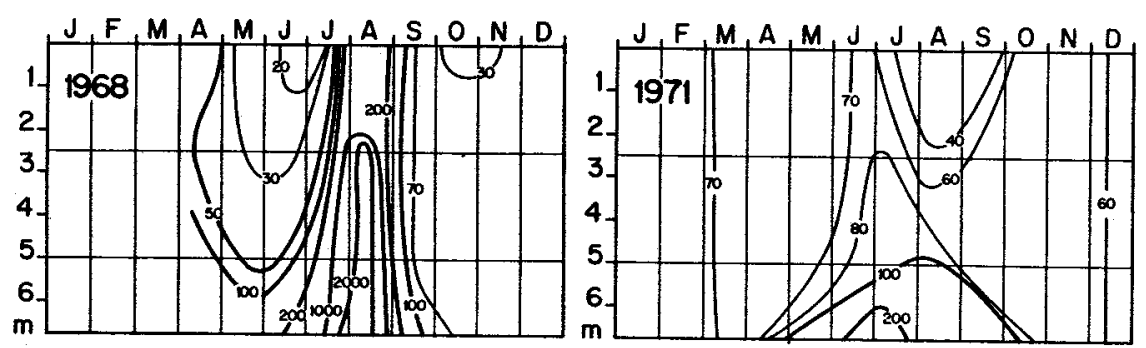

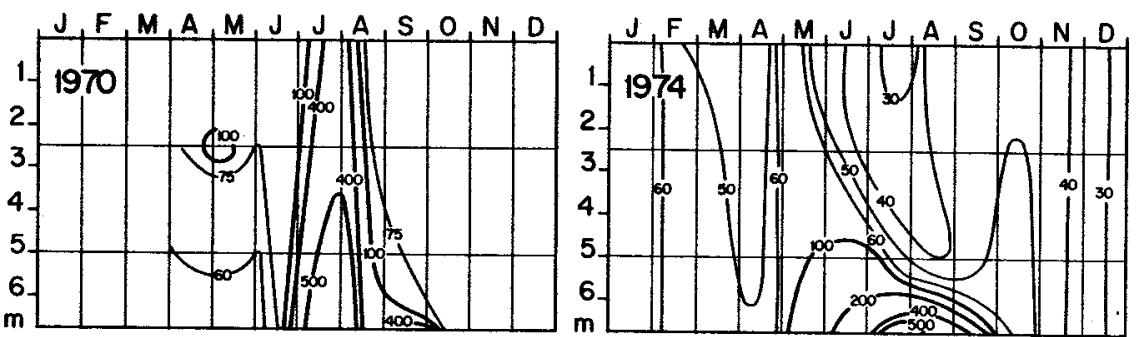

Abb. 5. Veränderung der Gesamtphosphorkonzentration in Abhängigkeit von der Jahreszeit und der Seetiefe. Angaben in $\mu \mathrm{g} P / 1$.

Fig. 5. Seasonal variation of total phosphorus concentration in the unfiltered samples. Concentrations expressed in $\mu \mathrm{g} \mathbf{P} / \mathrm{l}$.

waren es noch $484 \mu \mathrm{g}$ P/1, $197193 \mu \mathrm{g}$ P/l, 1974 konnte während der Sommerstagnation in dieser Tiefe kein Konzentrationsanstieg mehr beobachtet werden. Während der Herbstvollzirkulationen 1968 bis 1971 lag die Gesamtphosphorkonzentration praktisch unverändert bei etwa $70 \mu \mathrm{g}$ P/l. Im Oktober 1974 wurden nur noch $50 \mu \mathrm{g} \mathrm{P} / 1$, im Dezember sogar nur noch $35 \mu \mathrm{g}$ P/l beobachtet.

Stickstoff tritt in einem See in den Oxidationsstufen $+5,+3,+1,0,-1$ und -3 auf, die über verschiedene Reaktionswege ineinander übergeführt werden können [3] und je nach Tiefe und Jahreszeit in wechselndem Mengenverhältnis zueinander auftreten. Da es nicht das Ziel dieser Arbeit ist, den Stickstoffkreislauf des Sees zu beschreiben, sollen nur einige spezielle Punkte besonders herausgestellt werden.

Abb. 6 zeigt, dass im Zeitabschnitt 1968 bis 1971 die Gesamtstickstoffkonzentration während der Wintermonate stetig abgenommen hat. Im Gegensatz zum Phosphor, wo eine bedeutende Konzentrationsabnahme erst im Jahre 1974 beobachtet wurde, blieb im Falle des Gesamtstickstoffs die Konzentration im Zeitabschnitt 1971 bis 1974 praktisch unverändert. Maximale Gehalte an Gesamtstickstoff wurden alljährlich im Winter, minimale Werte während der Sommerstagnation beobachtet.

Nitrat kann sowohl anaerob (bakteriell) als auch aerob (durch Phytoplankton) reduziert werden oder aber durch Nitrifikationsprozesse aus Ammoniak aufoxidiert werden. Während 1968 im Juni und Juli sowie 1969 im Juli die Nitratkonzentration über das ganze Tiefenprofil unter der Nachweisbarkeitsgrenze lag, konnte seit 1970 Nitrat auch während der Sommerstagnation in allen Proben nachgewiesen werden (Tab. 1). Eingangs der Sommerstagnation ist die epilimnische Nitratzehrung kleiner

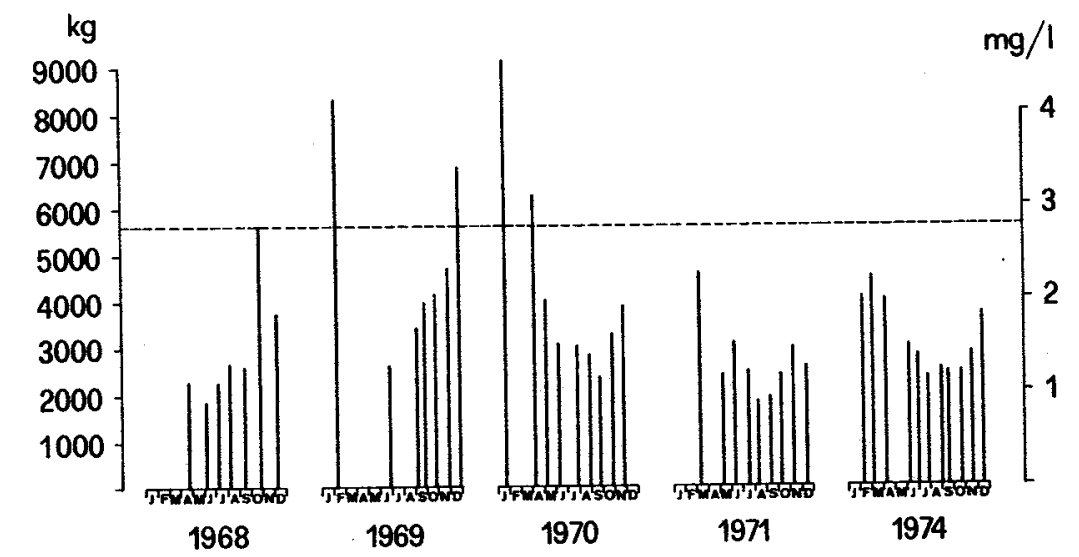

Abb.6. Gesamtstickstoffgehalt des Mauensees (kg) sowie mittlere Gesamtstickstofikonzentration (mg/l). Fig.6. Seasonal variations of total nitrogen (Kjeldahl-N $+\mathrm{NO}_{3}+\mathrm{NO}_{2}$ ) content $(\mathrm{kg})$ of the lake and the calculated mean concentration (content/lake volume). The dotted line represents the nitrogen concentration of the inflows during fall and winter overturn. Note that since 1971 the lake does no longer react as a nitrogen source during winter overturn.

geworden: 1969 wurden im Mittel 1,9 mg N/m ${ }^{3}$ Tag gezehrt, 1970 1,4 mg N/m ${ }^{3}$ Tag und 1971 und $1974 \mathrm{je} 0,4 \mathrm{mg} \mathrm{N} / \mathrm{m}^{3}$ Tag.

Die während der Sommerstagnation erhöhten Nitratkonzentrationen in der tropholytischen Schicht deuten in Übereinstimmung mit der erhöhten Sauerstoffspannung (siehe Abb.3) auf niedrigere Denitrifizierungs- oder aber auf erhöhte Nitrifikationsrate hin.

Die Primärproduktionsraten wurden nur in den Jahren 1971 und 1974 gemessen. In Abb. 7 wurden zudem die von ZimmermanN [23] gemessenen Werte dargestellt. Bei Berücksichtigung der Zufälligkeit der Probenahme unterscheiden sich die flächenspezifischen Jahresproduktionsraten von $347 \mathrm{~g} / \mathrm{m}^{2} \mathrm{Jahr}$ im Jahre 1971 und $303 \mathrm{~g} / \mathrm{m}^{2}$ Jahr im Jahre $1974 \mathrm{kaum}$. Die nach Angaben von Zimmermann berechneten Tagesproduktionsraten lagen während der Sommerstagnation 1967 nicht höher als die in den Jahren 1971 und 1974 bestimmten Werte. Wesentliche Unterschiede ergeben sich jedoch im vertikalen Produktionsprofil. Während 1967 maximale Produktionsraten durchwegs an der Seeoberfläche gemessen wurden, lag das Produktionsoptimum $\left(P_{\text {opt }}\right)$ in den Jahren 1971 und 1974 meist in etwa $1 \mathrm{~m}$ Tiefe. Es ist wahrscheinlich, dass das Produktionsoptimum 1967 zwischen der Oberfläche und der Tiefe von $1 \mathrm{~m}$ lag und von Zimmermann nicht beobachtet wurde. Die nach seinen Angaben berechneten Werte unterschätzen daher sehr wahrscheinlich die effektiven Tagesproduktionsraten. Während 1967 vor dem Einsetzen der Herbstteilzirkulation die Produktionsraten in $2 \mathrm{~m}$ Tiefe praktisch auf Null abfielen, wurde 1971 und 1974 in dieser Tiefe stets noch mindestens zwei Drittel des Wertes von $\boldsymbol{P}_{\text {opt }}$ beobachtet. Während der Sommerstagnation zeichnen sich demnach die Jahre 1971 und 1974 gegenüber 1967 aus durch ein niedrigeres, in grösserer Seetiefe liegendes $\boldsymbol{P}_{\text {opt }}$ und ein ausgeglicheneres Produktionsprofil, mit anderen Worten durch eine grössere Mächtigkeit der trophogenen Schicht. 
Tabelle 1. Nitratkonzentration ( $\mu \mathrm{g} \mathrm{N} / \mathrm{l})$.

Table 1. Nitrate concentrations $(\mu \mathrm{g} \mathrm{N} / \mathrm{l})$.

\begin{tabular}{rllllll}
\hline Tiefe & 6.Juni & 9.Juli & $\begin{array}{l}1968 \\
\text { 6.Aug. }\end{array}$ & 16.Sept. & 24.Okt. & 8.Dez. \\
\hline $0 \mathrm{~m}$ & 0 & 0 & 100 & 100 & 1400 & 300 \\
$2,5 \mathrm{~m}$ & 0 & 0 & 100 & 100 & 1400 & 300 \\
$5 \mathrm{~m}$ & 0 & 0 & 100 & 100 & 1300 & 300 \\
$6,5 \mathrm{~m}$ & 0 & 0 & 100 & 100 & 1400 & 300 \\
\hline
\end{tabular}

\begin{tabular}{rllllll} 
Tiefe & 12.Febr. & 25.Juni & $\begin{array}{c}1969 \\
1 . \text { Sept. }\end{array}$ & 23.Okt. & 24.Nov. & 22.Dez. \\
\hline $0 \mathrm{~m}$ & 2700 & 0 & 60 & 900 & 800 & 1600 \\
$2,5 \mathrm{~m}$ & 2800 & 0 & 90 & 750 & 800 & 2000 \\
$5 \mathrm{~m}$ & 2400 & 0 & 120 & 750 & 800 & 2100 \\
$6,5 \mathrm{~m}$ & 2300 & 0 & 60 & 650 & 800 & 2100 \\
\hline
\end{tabular}

\begin{tabular}{rllllll} 
Tiefe & 27.Jan. & 7.April & $\begin{array}{l}1970 \\
5 . \text { Mai }\end{array}$ & 2.Juni & 30.Juni & 28.Juli \\
\hline $0 \mathrm{~m}$ & 2700 & 1660 & 750 & 310 & 950 & 30 \\
$2,5 \mathrm{~m}$ & 2700 & 1660 & 750 & 570 & 950 & 30 \\
$5 \mathrm{~m}$ & 2600 & 1640 & 780 & 270 & 450 & 40 \\
$6,5 \mathrm{~m}$ & 2400 & 1660 & 800 & 90 & 50 & 40 \\
\hline
\end{tabular}

\begin{tabular}{rllllll}
\hline Tiefe & 24.Aug. & 25. Sept. & $\begin{array}{l}1970 \\
27 . \text { Okt. }\end{array}$ & 26. Nov. & & \\
\hline $0 \mathrm{~m}$ & 41 & 70 & 160 & 340 & & \\
$2,5 \mathrm{~m}$ & 38 & 60 & 150 & 340 & & \\
$5 \mathrm{~m}$ & 26 & 70 & 150 & 340 & & \\
$6,5 \mathrm{~m}$ & 8 & 70 & 140 & 340 & & \\
\hline & & & & & & \\
& & & 1971 & & & \\
Tiefe & $10 . \mathrm{Mäz}$ & 5. Mai & $6 . J$ uni & 15. Juli & 9. Aug. & 6. Sept. \\
\hline $0 \mathrm{~m}$ & 580 & 170 & 330 & 40 & 50 & 30 \\
$2,5 \mathrm{~m}$ & 590 & 160 & 500 & 140 & 20 & 20 \\
$5 \mathrm{~m}$ & 580 & 80 & 50 & 40 & 40 & 20 \\
$6 \mathrm{~m}$ & 600 & 60 & 60 & 40 & 40 & 30 \\
\hline
\end{tabular}

\begin{tabular}{rlll} 
Tiefe & 4. Okt. & 10.Nov. & $\begin{array}{l}1971 \\
9 . \text { Dez. }\end{array}$ \\
\hline $0 \mathrm{~m}$ & 40 & 120 & 280 \\
$2,5 \mathrm{~m}$ & 50 & 120 & 280 \\
$5 \mathrm{~m}$ & 30 & 120 & 280 \\
$6 \mathrm{~m}$ & 40 & 130 & 300 \\
\hline
\end{tabular}

Tabelle 1. Nitratkonzentration ( $\mu \mathrm{g} \mathrm{N} / \mathrm{l})$.

Table I. Nitrate concentrations ( $\mu \mathrm{g} \mathrm{N} / \mathrm{l})$.

\begin{tabular}{rllllll}
\hline Tiefe & 6.Febr. & 4.März & $\begin{array}{l}1974 \\
\text { 3.April }\end{array}$ & 30.April & 6.Juni & 25.Juni \\
\hline $0 \mathrm{~m}$ & 750 & 940 & 840 & 270 & 450 & 310 \\
$2,5 \mathrm{~m}$ & 780 & 890 & 840 & 320 & 400 & 300 \\
$5 \mathrm{~m}$ & 760 & 880 & 790 & 380 & 230 & 70 \\
$6,5 \mathrm{~m}$ & 770 & 900 & 660 & 240 & 80 & 40 \\
\hline & & & & & & \\
& & & 1974 & & & \\
Tiefe & 24. Juli & 21. Aug. & 11. Sept. & 15. Okt. & 12. Nov. & 11. Dez. \\
\hline $0 \mathrm{~m}$ & 100 & 110 & 40 & 160 & 410 & 660 \\
$2,5 \mathrm{~m}$ & 100 & 90 & 30 & 180 & 430 & 680 \\
$5 \mathrm{~m}$ & 100 & 90 & 20 & 170 & 420 & 670 \\
$6,5 \mathrm{~m}$ & 80 & 130 & 20 & 160 & 420 & 700 \\
\hline
\end{tabular}
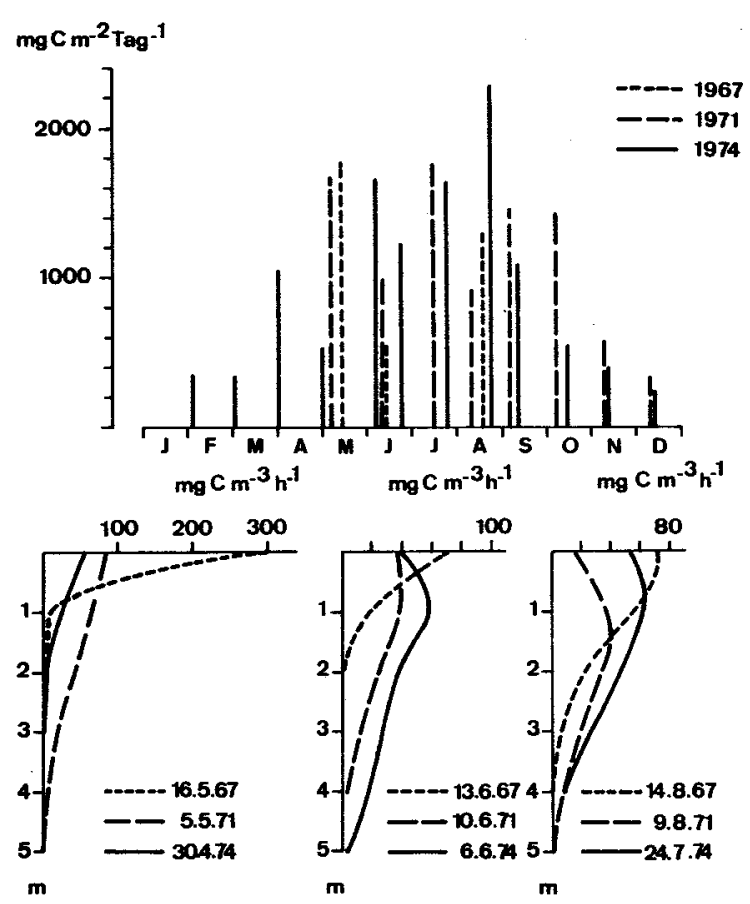

Abb. 7. Flächenspezifische Tagesproduktion in Abhängigkeit von der Jahreszeit (oben) und ausgewählte Produktionsprofile (unten).

Fig. 7. Seasonal variation of areal primary production rates (above) and selected primary production profiles (below). 
Das Phytoplankton wurde nur 1974 qualitativ-quantitativ analysiert (siehe Abb. 8). Ein Vergleich mit den drei Untersuchungen von ZIMMERManN [23] zeigt,

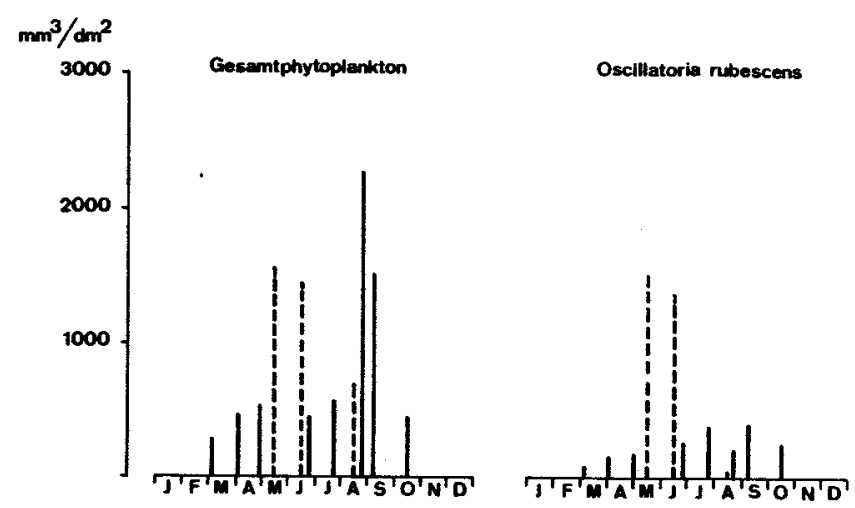

Abb. 8. Phytoplankton-Biomasse in Abhängigkeit von der Jahreszeit.

Fig. 8. Seasonal variation of total phytoplankton biomass (left) and Oscillatoria biomass (right).$$
\text { -- } 1967
$$

dass 1974 die Biomasse der Monate Mai bis Juni nur etwa einen Drittel des Wertes von 1967 erreichte. Die maximal beobachtete Oscillatoria-Biomasse sank von $1,5 \mathrm{~g} / \mathrm{dm}^{2}$ im Jahre 1967 auf $0,4 \mathrm{~g} / \mathrm{dm}^{2}$ im Jahre 1974 ab. Der Biomasseanstieg während der Monate August bis September 1974 ist auf ein gehäuftes Auftreten der sehr voluminösen Form Ceratium hirundinella (70000 $\mu^{3} /$ Zelle) zurückzuführen. Bezüglich der Artenzusammensetzung wurde festgestellt, dass 1974 neben Oscillatoria als weitere Blaualge Aphanizomenon flos-aque und Anabaena planctonica auftraten, zwei Formen, die von Zimmermann nicht beobachtet wurden. Aphanizomenon erreichte im August die gleiche Dichte wie Oscillatoria, Anabaena machte nie mehr als $5 \%$ des gesamten Blaualgenvolumens aus. Die 1967 stark vertretene Chrysophycee Dinobryon sp. wurde 1974 nur vereinzelt beobachtet.

\section{Diskussion}

Am Ableitungsrohr wurde im Zeitabschnitt 1968 bis 1975 baulich nichts verändert, und während der Sommerstagnation konnte alljährlich mit $Q_{\max }=4 \mathrm{~m}^{3} / \mathrm{min}$ gefahren werden. Die unterschiedlichen Temperaturverteilungen während der fünf Beobachtungsjahre sind daher nicht das Resultat unterschiedlicher Ableitungsbedingungen, sondern sie deuten darauf hin, dass während der Beobachtungsperiode die klimatischen Bedingungen nicht in allen Jahren identisch waren. Es ist daher das Ziel der Diskussion zu überprüfen, ob der beobachtete Trend einer Verbesserung des allgemeinen Seezustandes tatsächlich auf den Betrieb der Tiefenwasserableitung (TWA) zurückgeführt werden kann oder ob es sich dabei nur um eine witterungsbedingte Episode handelt.

Die Ableitung von kaltem, nährstoffreichem Hypolimnionwasser anstelle des natürlicherweise abfliessenden Oberflächenwassers beeinflusst einen See in mannigfacher Weise:
- Erhöhung des Energiegewinns während der Periode positiver Energiebilanz.

- Erosion des Hypolimnions.

- Verkürzung der Stagnationsdauer, sofern durch den Betrieb der TWA die Temperatur auch über dem Seegrund ansteigt.

- Erhöhung des Nährstoffexports aus dem See.

Unter den gegebenen klimatischen und topographischen Verhältnissen des Mauensees vermag sich während der Dauer der Stagnationsperiode kein eigentliches Hypolimnion auszubilden. Da in diesem Flachgewässer die Thermokline stets den Grund erreicht, kann etwas idealisiert nur zwischen zwei Schichten unterschieden werden, einem mehr oder weniger homothermen Epilimnion und einem thermisch stärker geschichteten Metalimnion. Die Grenze zwischen den beiden Zonen verläuft im Mittel in etwa $4 \mathrm{~m}$ Tiefe. Das Volumen des Metalimnions beträgt daher etwa $0,5 \cdot 10^{6} \mathrm{~m}^{3}$. Durch die verlegte Leitung werden dem See an seiner tiefsten Stelle während der Stagnationsperiode $10^{6} \mathrm{~m}^{3}$ Wasser entzogen. Daraus folgt, dass während der sechs Monate dauernden Stagnationsperiode das Wasser unterhalb einer Tiefe von $4 \mathrm{~m}$ etwa zweimal erneuert wird.

Die Beobachtung, dass im Bereich der Leitung die Schichtungsverhältnisse identisch sind mit denjenigen am andern Ende des Sees (siehe Abb.1), zeigt, dass die TWA den ganzen See erfasst und ihm am tiefsten Punkt Schicht um Schicht entzieht, ohne im Bereich des Auslaufes die Schichtung zu zerstören. Da die Isobathenfläche mit zunehmender Tiefe stetig kleiner wird, aus Gründen der Kontinuität aber der Wasserdurchsatz/Zeiteinheit durch jeden Horizont gleich gross sein muss, nimmt die Geschwindigkeit der abwärtsgerichteten Strömung mit wachsender Tiefe zu (Tab. 2).

Tabelle 2. Strömungsgeschwindigkeit als Funktion der Seetiefe.

Table 2 . Estimated velocity of the siphon induced downwards flow as a function of depth.

\begin{tabular}{llllll}
\hline $\begin{array}{l}\text { Tiefe } \\
\text { (depth) }\end{array}$ & (m) & 4 & 5 & 6 & 6,7 \\
$\begin{array}{l}\text { Geschwindigkeit } \\
\text { (velocity) }\end{array}$ & (m/Monat) & 0,6 & 0,9 & 1,6 & 12,6 \\
\hline
\end{tabular}

Die mittleren Geschwindigkeiten in den Schichten $4 \mathrm{~m}$ bis $5 \mathrm{~m}, 5 \mathrm{~m}$ bis $6 \mathrm{~m}$ und $6 \mathrm{~m}$ bis Seegrund betragen etwa $0,74 \mathrm{~m} /$ Monat, $1,15 \mathrm{~m} /$ Monat und $4 \mathrm{~m} /$ Monat. Daraus folgt, dass Wasserteilchen, die zur Zeit Null den 4 -m-Horizont passieren, nach 40 Tagen die Tiefe von $5 \mathrm{~m}$, nach 67 Tagen jene von $6 \mathrm{~m}$ erreichen und nach maximal 76 Tagen Aufenthaltszeit im Metalimnion den See via die Leitung verlassen. Bei einer Stagnationsdauer von etwa 180 Tagen wird daher die Aufenthaltszeit des Wassers im Metalimnion durch den Betrieb der TWA deutlich vermindert. Da die Sauerstoffkonzentration eines definierten Wasservolumens nicht nur von der Intensität der sauerstoffzehrenden Prozesse, sondern wesentlich von der Dauer seines Aufenthalts in der tropholytischen Zone beeinflusst wird, wird es klar, weshalb sich seit Inbetriebnahme der TWA die Sauerstoffverhältnisse im Metalimnion verbessert haben. Zusätzlich zu diesem Effekt der TWA lässt sich aufgrund der 
Primärproduktionsprofile vermuten, dass die trophogene Zone während der Sommerstagnation 1971 und 1974 zeitweise ins obere Metalimnion hineinreichte, im Sommer 1967 aber eindeutig auf die obersten 2 bis $3 \mathrm{~m}$ des Epilimnions beschränkt blieb. Dies bedeutet, dass während der Sommerstagnation 1967 in Tiefen von mehr als $4 \mathrm{~m}$ stets die sauerstoffzehrenden Prozesse 1971 und $1974 \mathrm{im}$ Tagesmittel zumindest zeitweise die sauerstoffproduzierenden Prozesse überwogen.

Die TWA beeinflusst daher die Sauerstoffkonzentration des Metalimnions in dreifacher Weise:

- Durch die Herabsetzung der Aufenthaltsdauer des Wassers im Metalimnion.

- Durch den biologischen Eintrag von Sauerstoff ins Metalimnion.

- Durch eine verringerte Belastung des Metalimnions mit autochthon produziertem organischem Material (siehe S. 23).

Um den Einfluss der TWA auf den Phosphorhaushalt des Sees beurteilen zu können, wurden in Tabelle 3 der Phosphorgehalt, die P-Belastung, der P-Export sowie die P-Austauschraten zwischen dem Sediment und dem Wasser abgeschätzt. Zur Berechnung des Phosphorgehalts $G_{\mathrm{P}}$ wurde der See in sieben Schichten unterteilt. Die Isobathenflächen $F_{Z}$ wurden in Ermangelung von exakten Messdaten nach der von IMBODEN [9] gegebenen Gleichung

$$
F(Z)=A_{0}\left[1-\frac{Z}{Z_{\max }}\right]^{\eta}
$$

approximiert, wobei $Z$ die Tiefe, $Z_{\max }$ die maximale und $\bar{Z}$ die mittlere Seetiefe und $A_{0}$ die Seeoberfläche bedeuten, und

$$
\eta=\frac{Z_{\max }}{\bar{Z}}-1
$$
Das Volumen $V\left(Z_{1}, Z_{2}\right)$ einer durch die Tiefen $Z_{1}$ und $Z_{2}$ begrenzten Schicht
ergibt sich aus

$$
\begin{gathered}
V\left(Z_{1}, Z_{2}\right)=\int_{Z_{1}}^{Z_{2}} F(Z) d z=\left.\left(\frac{-Z_{\max }}{\eta+1}\right) A_{0}\left[1-\frac{Z}{Z_{\max }}\right]^{\eta+1}\right|_{Z_{1}} ^{Z_{2}} \\
=-A_{0} \frac{Z_{\max }}{1+\eta}\left\{\left(1-\frac{Z_{2}}{Z_{\max }}\right)^{\eta+1}-\left(1-\frac{Z_{1}}{Z_{\max }}\right)^{\eta+1}\right\}
\end{gathered}
$$

Da nach (2) $\frac{Z_{\max }}{1+\eta}=\bar{Z}$

und $A_{0} \cdot \bar{Z}$ gleich dem totalen Seevolumen $V$ ist, folgt aus (2) und (3), dass

$$
V\left(Z_{1} ; Z_{2}\right)=V\left\{\left(1-\frac{Z_{1}}{Z_{\max }}\right)^{\eta+1}-\left(1-\frac{Z_{2}}{Z_{\max }}\right)^{\eta+1}\right\} \text {. }
$$

Der Phosphorgehalt des Sees wurde berechnet aus

$$
\begin{gathered}
G_{\mathrm{P}}=V(0 ; 0,63) \cdot\left[\mathrm{P}_{0}\right]+V(0,63 ; 1,88) \cdot \frac{\left[\mathrm{P}_{0}\right]+\left[\mathrm{P}_{2,5}\right]}{2}+V(1,88 ; 3,12)\left[\mathrm{P}_{2,5}\right] \\
+V(3,12 ; 4,38) \frac{\left[\mathrm{P}_{2,5}\right]+\left[\mathrm{P}_{5}\right]}{2}+V(4,38 ; 5,62)\left[\mathrm{P}_{5}\right]+V(5,62 ; 6,4)\left[\mathrm{P}_{6}\right] \\
+V(6,4 ; 6,8)\left[\mathrm{P}_{\mathrm{gr}}\right],
\end{gathered}
$$

wobei $\left[\mathbf{P}_{0}\right],\left[\mathbf{P}_{2,5}\right],\left[\mathbf{P}_{5}\right],\left[\mathbf{P}_{6}\right]$ und $\left[\mathbf{P}_{\mathrm{gr}}\right]$ die Gesamtphosphorkonzentration in den Tiefen $0 \mathrm{~m}, 2,5 \mathrm{~m}, 5 \mathrm{~m}, 6 \mathrm{~m}$ und über dem Seegrund bedeuten. $\left[\mathbf{P}_{0}\right],\left[\mathbf{P}_{2,5}\right]$ und $\left[P_{5}\right]$ sind stets gemessene Werte. $\left[P_{6}\right]$ wurde für die Jahre 1968, 1969, 1970 und 1974 ermittelt durch lineare Interpolation zwischen $\left[P_{5}\right]$ und $\left[P_{g r}\right]$. Für das Jahr 1971 wurde $\left[P_{g r}\right]$ durch lineare Extrapolation aus $\left[P_{5}\right]$ und $\left[P_{6}\right]$ berechnet.

Die Phosphorbelastung des Sees während des Zeitabschnitts $t_{1}$ bis $t_{2} L_{\mathrm{P}}\left(t_{1}, t_{2}\right)$ setzt sich zusammen aus einer konstanten Abwasserbelastung und einer variablen bodenbürtigen Belastung. Es wurde verschiedentlich gezeigt (2. B. [6, 10]), dass die bodenbürtige P-Fracht eines Vorfluters wenn nicht gar progressiv, dann zumindest linear mit zunehmendem Abfluss ansteigt.

Zur Abschätzung der P-Belastung des Sees im Zeitabschnitt $t_{1}$ bis $t_{2}$ wurde daher der folgende Ansatz gewählt

$$
L_{\mathrm{P}}\left(t_{1}, t_{2}\right)=\left(t_{2}-t_{1}\right)\left[K_{1}+K_{2} \cdot Q\left(t_{1}, t_{2}\right)\right],
$$

wobei unter $K_{1}$ die mittlere Abwasserbelastung pro Tag und unter $K_{2}$ die mittlere bodenbürtige P-Konzentration der Zuflüsse und $Q\left(t_{1}, t_{2}\right)$ die mittlere tägliche $\mathrm{Ab}$ flussrate verstanden wird. Für den speziellen Fall des Mauensees gilt

$K_{1}=\frac{209 \mathrm{~kg} \mathrm{P}}{365 \text { Tage }}=0,574 \mathrm{~kg} \mathrm{P} \mathrm{Tag}^{-1}$,

$K_{2}=\frac{4,3 \mathrm{~km}^{2} \cdot 35 \mathrm{~kg} \mathrm{P} \mathrm{km}^{-2} \mathrm{Jahr}^{-1}}{3,3 \cdot 10^{6} \mathrm{~m}^{3} \mathrm{Jahr}^{-1}}=45,6 \mathrm{mg} \mathrm{m}^{-3}$.

Der Phosphorexport $E_{\text {tot }}$ aus dem See setzt sich zusammen aus dem durch den natürlichen Abfluss und dem durch die Leitung ausgeschwemmten Phosphor

$$
E_{\text {tot }}=E_{0}+E_{\mathrm{TWA}} \text {, }
$$

wobei

$$
E_{0}\left(t_{1}, t_{2}\right)=\frac{\left[\mathrm{P}_{0}\left(t_{1}\right)\right]+\left[\mathrm{P}_{0}\left(t_{2}\right)\right]}{2} \cdot\left[Q\left(t_{1}, t_{2}\right)-Q_{\mathrm{TWA}}\right]\left(t_{2}-t_{1}\right)
$$

und

$$
E_{\mathrm{TWA}}\left(t_{1}, t_{2}\right)=\frac{\left[\mathrm{P}_{\mathrm{TWA}}\left(t_{1}\right)\right]+\left[\mathrm{P}_{\mathrm{TWA}}\left(t_{2}\right)\right]}{2} \cdot Q_{\mathrm{TWA}}\left(t_{2}-t_{1}\right)
$$

Die mittlere Abflussrate $Q$ ist Mitte Februar mit etwas über $5000 \mathrm{~m}^{3} /$ Tag minimal und erreicht Mitte Juli mit etwa $15000 \mathrm{~m}^{3} /$ Tag das Maximum. $Q_{\mathrm{TWA}}$ beträgt $5760 \mathrm{~m}^{3} /$ Tag und unter $\left[\mathrm{P}_{\text {TWA }}(t)\right]$ wird die zum Zeitpunkt $t$ im Auslauf der Leitung 
Tabelle 3. Phosphorbilanz des Sees.

Table 3. Phosphorus mass balance. $\left[\mathrm{P}_{\mathrm{TW}}\right]\left[\mathrm{P}_{0}\right]$ phosphorus concentration in the siphon and the lake surface, respectively. $G_{\mathrm{P}}$ total phosphorus content of the lake water. $Q$ flow rate, $\mathrm{m}^{3} /$ day. $E_{\mathrm{TWA}}, E_{0}$ phosphorus export by the siphon and natural surface outflow. respectively. $E_{\text {tot }}$ total phosphorus export. $L_{\mathrm{P}(\mathrm{AW})}$ and $L_{\mathrm{P}(\mathrm{LW})}$ phosphorus loading due to sewage and agriculture respectively. $L_{\mathrm{P}}$ total loading. $\Phi$ phosphorus net-flux to the sediments (if positive) or from the sediment to the water (if negative) estimated from (10). $\$$ mean daily flux rate. $\left[\mathrm{O}_{2}\right]$ oxygen concentration in the bottom overlaying water.

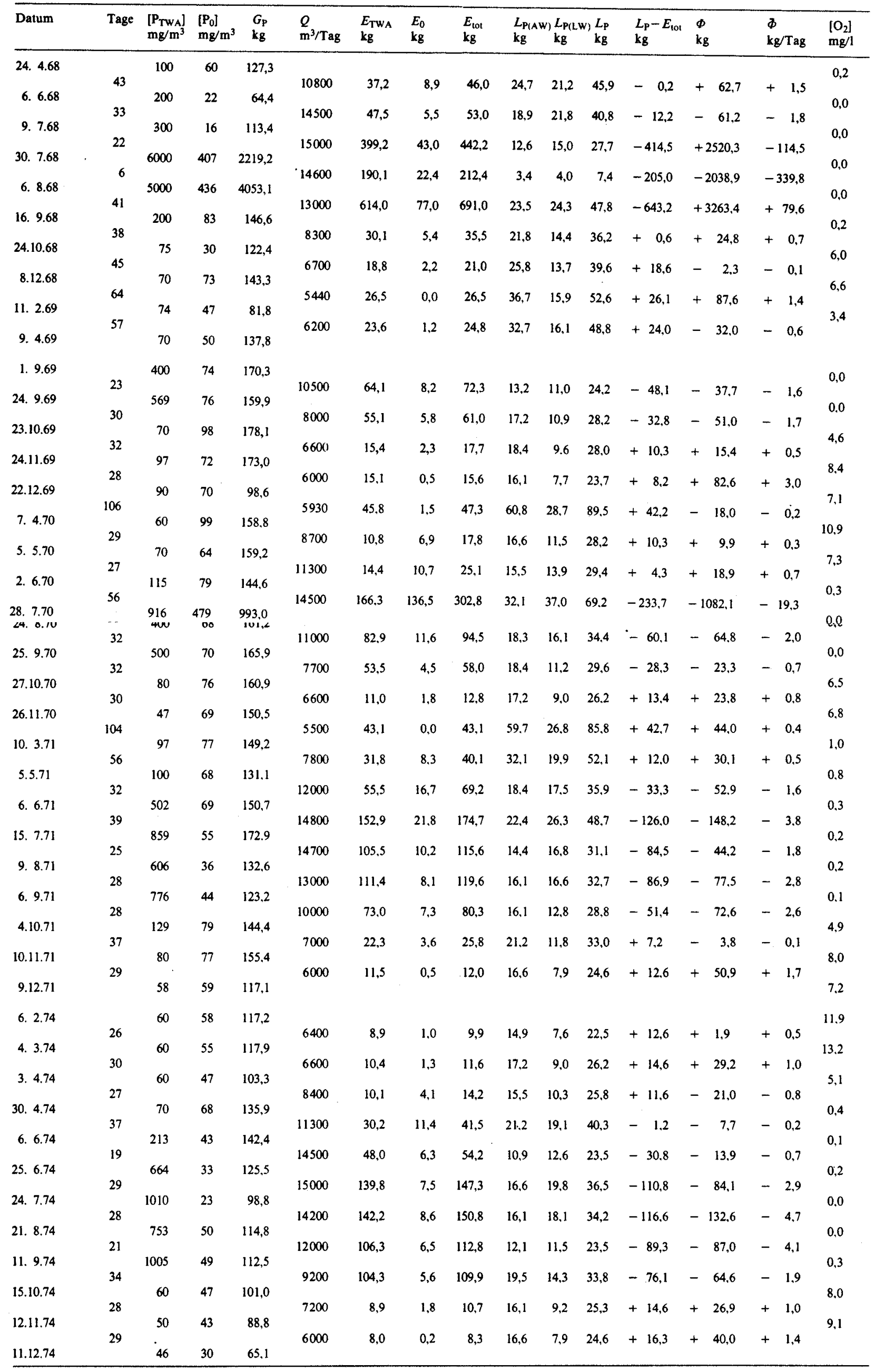


gemessene Gesamtphosphorkonzentration verstanden. Ist $Q_{\text {TWA }}$ im Februar grösser oder gleich $Q$, so wird der natürliche Abfluss und damit $E_{0}$ gleich Null gesetzt. Wenn bei Hochwasser die Leitung unter der Oberfläche des natürlichen Seeausflusses mündete, konnten aus der Leitung keine Proben erhoben werden. In diesen Fällen wurde in (9) $\left[P_{T W_{A}}\right]$ durch $\left[P_{g r}\right]$ ersetzt. Da $\left[P_{g r}\right] \leq\left[P_{T W_{A}}\right]$, wurde in Tabelle 3 der Phosphorexport aus dem See zeitweise unterschätzt. Der Nettophosphorfluss $\Phi_{\mathrm{P}}$ vom Wasser zum Sediment oder vice versa ergibt sich aus

$$
G_{\mathrm{P}}\left(t_{2}\right)=G_{\mathrm{P}}\left(t_{1}\right)+L_{\mathrm{P}}\left(t_{1}, t_{2}\right)-E_{\mathrm{tot}}\left(t_{1}, t_{2}\right)-\Phi_{\mathrm{P}}\left(t_{1}, t_{2}\right)
$$

$\Phi_{\mathrm{P}}$ ist positiv, wenn es sich um einen Nettofluss vom Wasser zum Sediment handelt. Die mittlere, tägliche Sedimentations- oder Rücklösungsrate $\Phi\left(t_{1}, t_{2}\right)$ im Zeitabschnitt $t_{1}$ bis $t_{2}$ ist

$$
\Phi_{\mathrm{P}}\left(t_{1}, t_{2}\right)=\frac{\Phi_{\mathrm{P}}}{\left(t_{2}-t_{1}\right)}
$$

Die Bedeutung der Sedimente für den Phosphorhaushalt des Sees ist aus Tabelle 3 klar ersichtlich. War die Sauerstoffkonzentration über dem Seegrund im Mittel der Beobachtungsperiode niedriger als $1 \mathrm{mg} \mathrm{O} \mathrm{O}_{2} / 1$, so wurde in $91 \%$ aller Fälle ein Nettophosphorfluss von den Sedimenten zum Wasser beobachtet. War die Sauerstoffkonzentration dagegen im Mittel höher als $3 \mathrm{mg} \mathrm{O} \mathrm{O}_{2} /$, so reagierten die Sedimente in $95 \%$ der Fälle als «sink» für den Gesamtphosphor.

Im Sommer 1968 gaben die Sedimente während einer Periode von 28 Tagen etwa $4560 \mathrm{~kg} \mathrm{P}$ ans Wasser ab, wovon in einer anschliessenden Periode von 41 Tagen wieder $3270 \mathrm{~kg} P$ ausgefällt oder an der Sedimentoberfläche adsorbiert wurden. Dieses Wechselspiel von Rücklösung und Resedimentation führt zu einer ausgeprägten Phosphorakkumulation in der allerobersten Sedimentschicht. Im Falle des Mauensees muss sie 1968 mindestens $5000 \mathrm{~kg}$ an anaerob sehr leicht löslichen Phosphorverbindungen enthalten haben, was der vierzehnfachen jährlichen externen Phosphorzufuhr entspricht. Unter dieser Voraussetzung ist primär nicht die sedimentinterne Diffusion der geschwindigkeitsbegrenzende Faktor der Phosphorrücklösung, sondern die Rücklösung wird hauptsächlich kontrolliert durch die an der Sedimentoberfläche vorhandene Menge an löslichen Verbindungen, die Lösungsgeschwindigkeit, die Lage des Löslichkeitsgleichgewichts (Sättigungskonzentration) und die Diffusionsbedingungen im überstehenden Wasser.

Dies erklärt, abgesehen von Unterschieden in der Beschaffenheit der Sedimente, weshalb in Experimenten mit homogenen Sedimenten nie derart hohe Phosphorrücklösungsraten beobachtet werden. Die im Mauensee bestimmten maximalen Rücklösungsraten (siehe Tab. 3) betrugen $1968340 \mathrm{~kg}$ P/Tag ( $\left.=1000 \mathrm{mg} \mathrm{P} / \mathrm{m}^{2} \mathrm{Tag}\right)$ $197020 \mathrm{~kg} \mathrm{P} / \mathrm{Tag}$ ( $=70 \mathrm{mg} \mathrm{P} / \mathrm{m}^{2} \mathrm{Tag}$ ), 1971 und $19744 \mathrm{bis} 5 \mathrm{~kg}$ P/Tag (=13 bis $16 \mathrm{mg} \mathrm{P} / \mathrm{m}^{2} \mathrm{Tag}$ ). Diese Angaben bedeuten Nettorücklösungsraten. Die effektiven Bruttorücklösungsraten wären um den Betrag der Sedimentation höher. Untersuchungen von TESSENOW [21] mit homogenem Sedimentmaterial aus dem Ursee ergaben unter anaeroben Bedingungen maximale Rücklösungsraten von nur 10 bis $16 \mathrm{mg} \mathrm{P} / \mathrm{m}^{2} \mathrm{Tag}$. In eigenen Experimenten mit Vierwaldstätterseesedimenten lagen sie im Bereich von $14 \mathrm{mg} \mathrm{P} / \mathrm{m}^{2}$ Tag.
Der Prozess der Phosphorakkumulation in der obersten Sedimentschicht und der Auteutrophierung eines Sees kann folgendermassen verstanden werden: Ist die externe Phosphorbelastung eines Sees konstant und so niedrig, dass die Sedimentwasserkontaktzone ganzjährig aerob bleibt, so ist auch die pro Jahr sedimentierte und ins Sediment eingebaute Phosphormenge $S_{1}$ konstant, und die Rücklösungsrate kann gleich Null gesetzt werden. Unter diesen Voraussetzungen enthält die oberste Sedimentschicht im Vergleich zur entsprechenden Vorjahresschicht stets gleichviel Phosphor. Wird die externe Belastung so erhöht, dass sich im Hypolimnion anaerobe Bedingungen einstellen, so bewirkt dies einerseits, dass die neue Sedimentationsrate $S_{2}$ grösser wird als $S_{1}$, und anderseits, dass eine bestimmte Menge $M_{1}$ ins Hypolimnion rückgelöst wird. Treten im Herbst wieder aerobe Bedingungen ein, so wird zusätzlich zu $S_{2}$ auch ein Teil von $M_{1}$ wieder sedimentiert, so dass die neue jährliche Sedimentationsrate $S_{2}+k M_{1}$ beträgt. Dadurch steigt der Phosphorgehalt in der obersten Sedimentschicht an. Ist nun die Rücklösung abhängig von der in der obersten Schicht vorhandenen löslichen Phosphormenge, so wird im darauffolgenden Sommer die rückgelöste Menge $M_{2}$ grösser sein als $M_{1}$ und die bis zum Winter aussedimentierende Menge $k M_{2}+S_{2}$ betragen. Da aber $k M_{2}+S_{2}$ grösser ist als $k M_{1}+S_{2}$, wird die Rücklösung im nächsten Sommer wiederum grösser sein als $M_{2}$ usw. Mit anderen Worten bedeutet dies, dass ein See sich selbst zu eutrophieren beginnt, sobald die externe Belastung soweit ansteigt, dass sich im Hypolimnion anaerobe Verhältnisse einstellen. Die Geschwindigkeit dieser Auteutrophierung ist um so grösser, je mehr des rückgelösten Phosphors durch Eddy-Diffusion in die trophogene Zone gelangt. Es ist daher anzunehmen, dass sich flache Seen rascher auteutrophieren als tiefe Gewässer. Es ist auch anzunehmen, dass die Auteutrophierung bei konstanter externer Belastung solange fortschreitet, bis der Phosphorexport im Jahresmittel gleichgross wird wie die externe Phosphorzufuhr oder bis sich während der anaeroben Phase über dem Seegrund P-Konzentrationen einstellen, die nahe bei der Sättigungskonzentration liegen und die Phosphorrücklösung nur noch von der Eddy-Diffusion im Hypolimnion, aber nicht mehr vom Phosphorangebot im Sediment beeinflusst wird. Bedingt durch die Verknüpfung des Eisen-Phosphor-Kreislaufs mit dem Eisen-Schwefel-Kreislauf ist es möglich, dass der Auteutrophierungsprozess dadurch beschleunigt wird, dass unter sehr stark reduzierenden Bedingungen zweiwertiges Eisen als Sulfid ausgefällt wird und dass rückgelöstes Phosphat beim Eintritt der Vollzirkulation nicht mehr abiotisch ausgefallt werden kann [12].

Der Auteutrophierung, die bewirkt, dass der Phosphorgehalt und die pro Jahr in einem See umgesetzte Phosphormenge auch bei konstanter externer Belastung ansteigt, kann auf verschiedenen Wegen entgegengewirkt werden:

- direkt, durch Erhöhung des Phosphorexports aus dem See,

- indirekt, durch Massnahmen, die dazu führen, dass im Hypolimnion die Sauerstoffkonzentration nie unter einen bestimmten Grenzwert, z. B. $3 \mathrm{mg} \mathrm{O} / 1$ (siehe S. 16), absinkt, sei dies nun durch Reduktion der externen Zufuhr oder durch die Belüftung des Hypolimnions.

Die Erklärung für die Verringerung der sommerlichen Phosphorrücklösung im Mauensee seit 1968 ist darin zu suchen, dass seit der Inbetriebnahme der TWA 
alljährlich mehr Phosphor aus dem See abfloss, als ihm zugeführt wurde. Für die Perioden vom 24.4.68 bis 9.4.69, 24.11.69 bis $26.11 .70,26.11 .70$ bis 9.12 .71 und 6.2 .74 bis 11.12 .74 wurden Exportüberschüsse von $1200 \mathrm{~kg}, 380 \mathrm{~kg}, 31 \mathrm{~kg}$ und $360 \mathrm{~kg}$ Phosphor berechnet. Seit der Inbetriebnahme der TWA wurden dem See insgesamt etwa $3700 \mathrm{~kg}$ mehr Phosphor entzogen als zugeführt. Da sich die Phosphorkonzentration während der Zirkulationsperioden 1968, 1969, 1970 und 1971 kaum verändert hat, muss der Exportüberschuss zwangsläufig zu einer Phosphorverarmung der Sedimentoberfläche geführt haben, was die rückläufige Entwicklung der P-Rücklösung während der Stagnationsperioden erklärt.

Während der Stagnationsperiode rückgelöster Phosphor, der nicht aus dem See ausgeschwemmt wird, wird bei Zutritt von Sauerstoff rasch wieder in den Sedimenten fixiert und beeinflusst die Phosphorkonzentration in der anschliessenden Zirkulationsphase nicht wesentlich. Da während der Zirkulationsphase $\left[\mathbf{P}_{0}\right]=\left[\mathbf{P}_{\text {rWA }}\right]$, ist eine Beeinflussung der P-Konzentration während der Zirkulation durch die
TWA unmöglich.

Die im Vergleich zu den Vorjahren deutlich niedrigere Gesamtphosphorkonzentration im Herbst/Winter 1974 ist damit zu erklären, dass seit dem Spätsommer 1974 die Abwässer aus dem Raum Hitzligen (St. Erhard) nicht mehr in den See eingeleitet werden. Gemäss Angaben von GÄCHTER [5] verringerte sich dadurch die P-Belastung von $0,86 \mathrm{~kg}$ P/Tag um $0,28 \mathrm{~kg}$ P/Tag auf $0,58 \mathrm{~kg}$ P/Tag oder $68 \%$ der ursprünglichen Belastung. Unter der Voraussetzung, dass die Phosphorsedimentation sich proportional verhalte zur Gesamtphosphorkonzentration im See,
folgt aus der Steady-state-Bedingung

Zufuhr $=$ Sedimentation + Export

$$
\begin{gathered}
Q \cdot\left[\mathrm{C}_{\mathrm{Z}}\right]=K \cdot[\mathrm{C}]+Q \cdot[\mathrm{C}] \\
{[\mathrm{C}]=\frac{Q \cdot\left[\mathrm{C}_{\mathrm{Z}}\right]}{Q+K},}
\end{gathered}
$$

dass zwischen der Steady-state-Konzentration $[C]$ und der externen Belastung $Q \cdot\left[C_{z}\right]$ ein linearer Zusammenhang besteht. Vor der Fernhaltung der Abwässer von Hitzligen betrug die Gesamtphosphorkonzentration während der Vollzirkulation etwa $70 \mathrm{mg} \mathrm{P} / \mathrm{m}^{3}$. Nach (13) wäre für 1974 eine solche von $0,68 \cdot 70 \mathrm{mg} \mathrm{P} / \mathrm{m}^{3}$ oder $48 \mathrm{mg} \mathrm{P} / \mathrm{m}^{3}$ zu erwarten, was sehr gut mit dem tatsächlich beobachteten Wert von 30 bis $50 \mathrm{mg} \mathrm{P} / \mathrm{m}^{3}$ übereinstimmt.

Um beurteilen zu können, wieweit während der Sommerstagnation die seeinterne Nährstoffbelastung die trophogene Zone zu beeinflussen vermag, wurde analog zu (10) für die oberste Wasserschicht von $4,4 \mathrm{~m}$ Mächtigkeit eine Phosphorbilanz aufgestellt.

$$
G_{\mathrm{P}}\left(t_{2}\right)=G_{\mathrm{P}}\left(t_{1}\right)+L_{\mathrm{P}}\left(t_{1}, t_{2}\right)+R\left(t_{1}, t_{2}\right)-E_{0}\left(t_{1}, t_{2}\right)-S\left(t_{1}, t_{2}\right),
$$

wobei $R\left(t_{1}, t_{2}\right)$ die Rückdiffusion aus dem tieferliegenden Wasserkörper und $S\left(t_{1}, t_{2}\right)$ die Phosphorsedimentation aus der uptersuchten Wasserschicht heraus
bedeutet.
Nach GäCHTER [7] liegt die Sedimentationsgeschwindigkeit von partikulärem Phosphor in der Grössenordnung von $0,2 \mathrm{~m} / \mathrm{Tag}$. Da die mittlere Tiefe der untersuchten Wasserschicht etwa 3,3 m beträgt, gehen ihr täglich $6,2 \%$ ihres Gehalts an partikulärem Phosphor (PP) durch Sedimentation verloren. Daraus folgt

$$
S\left(t_{1}, t_{2}\right)=\frac{G_{\mathrm{PP}}\left(t_{1}\right)+G_{\mathrm{PP}}\left(t_{2}\right)}{2} \cdot 0,062 \cdot\left(t_{2}-t_{1}\right)
$$

Gleichung (15) in (14) eingesetzt und (14) nach $R$ aufgelöst, ergibt die in Tabelle 4 zusammengestellten Werte für die seeinterne Phosphorbelastung der trophogenen Schicht. Tabelle 4 zeigt, dass die seeinterne Phosphorbelastung der trophogenen Schicht zur Zeit der maximalen Nettorücklösung aus den Sedimenten die externe Belastung 1968 um etwa den dreihundertfachen Betrag übertraf. 1970 war die Rückdiffusion 32mal, 1971 5mal und 1974 noch $3 \mathrm{mal}$ so gross wie die externe Belastung.

Úbertrifft die Rückdiffusion die Sedimentationsverluste, so übertrifft die epilimnische Phosphorkonzentration diejenige der Zuflüsse, und der Phosphorexport wird auch ohne TWA grösser als die Phosphorzufuhr. Die mittlere Phosphorkonzentration des dem See zufliessenden Wassers beträgt während der Sommermonate etwa $85 \mathrm{mg} \mathrm{P} / \mathrm{m}^{3}$. Die im Epilimnion beobachteten Konzentrationsmaxima lagen 1968 und 1970 mit mehr als $400 \mathrm{mg} \mathrm{P} / \mathrm{m}^{3}$ deutlich über diesem Wert. Dies zeigt, dass eutrophe Flachseen nicht ganzjährig als Phosphorfalle wirken, sondern dass während der Sommerstagnation der Phosphorexport die Phosphorzufuhr zum See deutlich übertreffen kann.

Tabelle 4. Phosphorbilanz für die Wasserschicht 0 bis $4,4 \mathrm{~m}$ während der Zeit maximaler Phosphorrücklösung aus den Sedimenten.

Table 4. Phosphorus mass balance of the epilimnion (layer between lake surface and $4.4 \mathrm{~m}$ depth)

\begin{tabular}{|c|c|c|c|c|c|c|c|c|}
\hline Datum & Tage & $\begin{array}{l}G\left(\mathbf{P}_{\mathrm{tot}}\right) \\
\mathrm{kg}\end{array}$ & $\begin{array}{l}G(\mathrm{PP}) \\
\mathbf{k g}\end{array}$ & $\begin{array}{l}L\left(t_{1}, t_{2}\right) \\
\mathbf{k g}\end{array}$ & $\begin{array}{l}E\left(t_{1}, t_{2}\right) \\
\mathbf{k g}\end{array}$ & $\begin{array}{l}S\left(t_{1}, t_{2}\right) \\
\mathbf{k g}\end{array}$ & $\begin{array}{l}R\left(t_{1}, t_{2}\right) \\
\mathbf{k g}\end{array}$ & $\begin{array}{l}R \\
\mathrm{~kg} / \mathrm{Tag}\end{array}$ \\
\hline 9.7 .68 & & 65,7 & 52,5 & & & & & \\
\hline & 22 & & & 27,7 & 43,0 & 520,3 & 1885,2 & 85,7 \\
\hline 30.7 .68 & 6 & 1415,3 & 710,4 & 7,4 & 22,4 & 660,0 & 2164,4 & 360,7 \\
\hline 6.8 .68 & & 2904,7 & 2838,5 & & & & & \\
\hline 2.6.70 & 56 & 124,8 & 101,2 & 69,2 & 136,5 & 1441,4 & 2196,9 & 39,2 \\
\hline 28.7 .70 & & 813,0 & 729,1 & & & & & \\
\hline 6.6 .71 & 39 & 116,4 & 96,0 & 48,7 & 21,8 & 261,4 & 250,9 & 6,4 \\
\hline 15.7 .71 & & 132,8 & 120,2 & & & & & \\
\hline 24.7 .74 & 28 & 54,0 & 48,7 & 34,2 & 8,6 & 99,8 & 101,1 & 3,6 \\
\hline 21.8 .74 & & 80,9 & 66,3 & & & & & \\
\hline
\end{tabular}
Table 4. Phosphorus mass balance of the epilimnion (layer between lake $G\left(\mathrm{P}_{100}\right)$ total phosphorus during the periode of maximum phosphorus dissolution at the sediment surface. $G\left(\mathbf{P}_{\text {to }}\right)$ todil phosphort $S$ sedimentation, $R$ backdiffusion rate to the epilimnion calculated from (14) and (15). 
Bedingt durch mögliche Fehler bei der chemischen Analytik und die in (14) und (15) getroffenen vereinfachenden Annahmen kann der effektive Wert der seeinternen Belastung zwar vom berechneten Wert $R\left(t_{1}, t_{2}\right)$ etwas abweichen, sicher ist er aber in der Grössenordnung richtig. Tabelle 4 zeigt daher drei wesentliche Punkte:

1. In eutrophen Flachgewässern kann die seeinterne Belastung der trophogenen Zone die externe Nährstoffbelastung um ein Vielfaches übertreffen.

2. Die Rückdiffusion von Phosphor ins Epilimnion wurde seit der Inbetriebnahme der TWA alljährlich kleiner, trotz der flacheren Temperaturverteilung in den Jahren 1971 und 1974.

3. Bei der Beurteilung der trophischen Verhältnisse des Sees kann sie auch im Jahre 1974 noch nicht vernachlässigt werden.

Zusammengefasst beeinflusst die TWA den Phosphorhaushalt des Sees also dadurch, dass während der Sommerstagnation rückgelöster Phosphor nicht im See bleibt und bei Zutritt von Sauerstoff wieder sedimentiert wird, sondern dass er zum grossen Teil durch die TWA in den Seeausfluss gelangt. Dieser Prozess bewirkt eine Reduktion des Phosphorgehalts der Sedimente, eine kleinere Rücklösungsrate unter anaeroben Bedingungen und eine Abnahme der Rückdiffusion von Phosphorverbindungen aus der Sediment-Wasser-Kontaktzone ins Epilimnion.

Im Gegensatz zum Phosphor, wo sich wegen der Rücklösung aus den Sedimenten maximale Gehalte während der Sommerstagnation einstellten, erreichte der Gesamtstickstoff jeweils während der Zirkulationsphase das Jahresmaximum (siehe Abb.6). Da beim Stickstoffhaushalt eines Sees nicht nur die Wechselwirkungen mit dem Sediment, sondern auch jene mit der Atmosphäre in Betracht zu ziehen sind (N-Fixierung durch Bakterien und Blaualgen und Denitrifikationsprozesse), können Defizite oder Überschüsse in der Bilanz (10) nicht einfach mit Fixierung oder Mobilisierung von Stickstoffverbindungen an der Sedimentoberfläche erklärt werden. Der vollständige Ansatz für die Gesamtstickstoffbilanz

$$
\frac{d G_{\mathrm{N}}}{d t}=Z+F+R-(S+D+E)
$$

ist im allgemeinen Fall nicht lösbar, da $F, R, S$ und $D$ unbekannt sind. $G_{\mathrm{N}}, Z, F, R$, $S, D, E$ bedeuten die im See vorhandene Menge, Zufuhr via die Zuflüsse, Fixation aus der Atmosphäre, Rücklösung aus den Sedimenten, Sedimentation, Denitrifikation und Export via den Abfluss und die TWA.

Die mittlere Gesamtstickstoffkonzentration der Zuflüsse, herrührend aus dem Boden und dem Abwasser, beträgt nach GäCHTER [5, 6]

$$
\mathrm{C}_{Z}=\frac{4,3 \mathrm{~km}^{2} \cdot 2000 \mathrm{~kg} / \mathrm{km}^{2} \mathrm{Jahr}+711 \mathrm{~kg}}{3,3 \cdot 10^{6} \mathrm{~m}^{3} / \mathrm{Jahr}}=2,8 \mathrm{mg} / 1
$$

Ist $C>C_{Z}$, wie z. B. in den Wintern 1968/69 und 1970/71 (siehe Abb.6), so muss eine zusätzliche Stickstoffquelle in Betracht gezogen werden. Da bei den $\mathrm{zu}$ diesem Zeitpunkt herrschenden Konzentrationen von anorganischen Stickstoffverbin- dungen $\left(\left[\mathrm{NO}_{3}\right]=2,7 \mathrm{mg} \mathrm{N} / \mathrm{l}\right) F$ gleich Null gesetzt werden kann und wahrscheinlich auch die Denitrifikation unbedeutend ist, vereinfacht sich (16) zu

$$
\frac{d G_{\mathrm{N}}}{d t}=Z+R-(S+E)
$$

oder

$$
R-S=\frac{d G_{\mathrm{N}}}{d t}-Z+E .
$$

Da $d G_{N} / d t, Z$ und $E$ bekannt sind, lässt sich der Term $(R-S)$ für diesen Zeitabschnitt berechnen.

Tabelle 5 zeigt, dass die Sedimente zu Beginn der Herbst-Winter-Vollzirkulation praktisch ausnahmslos als Stickstoffquelle reagieren $([R-S]>0)$. Die Nettobelastung des Sees mit Stickstoffverbindungen aus den Sedimenten ist seit dem Winter 1970/71 stark zurückgegangen. Im Winter 1969/70 erreichte sie zeitweise mehr als den vierfachen Betrag der externen Belastung. Während der Herbst- und Wintermonate 1970, 1971 und 1974 betrug dagegen in der Regel die interne Belastung nur noch einen Bruchteil der externen Zufuhr.

Tabelle 5. Stickstoffbilanz im Herbst und im frühen Winter.

\begin{tabular}{|c|c|c|c|c|c|c|c|c|}
\hline $\begin{array}{l}\text { Datum } \\
\text { Date }\end{array}$ & $\begin{array}{l}t \\
\text { days }\end{array}$ & $\begin{array}{l}Q \\
\mathbf{m}^{3 / \text { day }}\end{array}$ & $\begin{array}{l}G_{\mathrm{N}} \\
\mathrm{kg}\end{array}$ & $\begin{array}{l}\Delta G_{\mathrm{N}} / \Delta t \\
\mathrm{~kg} / \mathrm{day}\end{array}$ & $\begin{array}{l}Z \\
\mathbf{k g} / \text { day }\end{array}$ & $\begin{array}{l}E \\
\mathrm{~kg} / \text { day }\end{array}$ & $\begin{array}{l}(Z-E) \\
\mathrm{kg} / \text { day }\end{array}$ & $\begin{array}{l}R-S= \\
\Delta G_{\mathrm{N}} / \Delta t-(Z-E) \\
\mathrm{kg} / \text { day }\end{array}$ \\
\hline 23.10 .69 & & & 4135 & & & & & \\
\hline 24.11 .69 & 32 & 6600 & 4743 & 19,0 & 19,1 & 14,6 & 4,5 & 14,5 \\
\hline 22.12 .69 & 28 & 6000 & 6811 & 73,9 & 17,5 & 17,3 & 0,2 & 73,6 \\
\hline & 36 & 6000 & & 64,2 & 17,5 & 23,9 & $-6,4$ & 70,5 \\
\hline $\begin{array}{l}27.1 .70 \\
27.10 .70\end{array}$ & & & $\begin{array}{l}9132 \\
3284\end{array}$ & & & & & \\
\hline & 30 & 6600 & & 20,3 & 19,1 & 11,8 & 7,2 & 13,0 \\
\hline 26.11 .70 & 104 & 5500 & 3892 & 7,0 & 16,2 & 11,7 & 4,5 & 2,5 \\
\hline 10. 3.71 & & & 4622 & & & & & \\
\hline 4.10 .71 & & & 2432 & & & & & \\
\hline 10.11 .71 & 37 & 7000 & & 15,8 & 20,1 & 9,5 & 10,6 & 5,2 \\
\hline & 29 & 6000 & & $-15,9$ & 17,5 & 8,3 & 9,2 & $-25,1$ \\
\hline $\begin{array}{r}9.12 .71 \\
15.1074\end{array}$ & & & 2554 & & & & & \\
\hline 15.10 .74 & 28 & & 2432 & & & & 112 & 18 \\
\hline 12.11 .74 & & 7200 & 2797 & 13,0 & 20,7 & 9,4 & 11,2 & 1,8 \\
\hline 11.12 .74 & 29 & 6000 & 3770 & 33,6 & 17,5 & 9,8 & 7,7 & 25,9 \\
\hline
\end{tabular}

Table 5 . Nitrogen mass balance in fall and early winter. $Q$ (daily flow rate), $G_{\mathrm{N}}$ nitrogen content, $Z$ (daily external nitrogen supply), $E$ (daily nitrogen export from the lake), $R$ (daily nitrogen release from the sediments), $S$ (nitrogen sedimentation rate). 
Die Stickstoffrücklösung aus den Sedimenten ist abhängig vom N-Gehalt in der Sedimentoberfläche und einer Reihe von Begleitparametern wie z. B. der Bakterienaktivität im Sediment, der Temperatur, dem Ammoniumgehalt im überstehenden Wasser usw.

$$
R=[\mathrm{PN}] \cdot F(l, m, n, \ldots)
$$

Da es keinen Grund gibt anzunehmen, dass sich seit der Inbetriebnahme der TWA diese Parameter während der Zirkulationsperiode verändert haben, kann die verringerte $N$-Rücklösung nur mit einem verringerten Stickstoffangebot an der Sedimentoberfläche erklärt werden.

In Abb.9 wird schematisch gezeigt, wie die TWA während der Stagnationsperiode den Stickstoffkreislauf des Sees und den Stickstoffgehalt der Sedimente beeinflusst. Durch Ammonifikation wird im Sediment $\mathrm{NH}_{4}^{+}$freigesetzt, das dem Konzentrationsgradienten folgend ins überstehende Wasser diffundiert. Die TWA bewirkt eine ständige Erneuerung des über der Sedimentoberfläche liegenden Wassers. Dies hat zur Folge, dass die Ammoniumkonzentration im Kontaktwasser herabgesetzt wird, was dank dem grösseren Konzentrationsgradienten eine beschleunigte Diffusion vom Sediment zum Wasser ermöglicht. Zusätzlich zu dieser rein physikalischen Wirkung der TWA erlaubt die durch sie bewirkte höhere Sauerstoffkonzentration über der Sedimentoberfläche erhöhte Nitrifikationsraten. Dieser Prozess verringert die $\left[\mathrm{NH}_{4}\right]$-Konzentration im Wasser auf chemischem

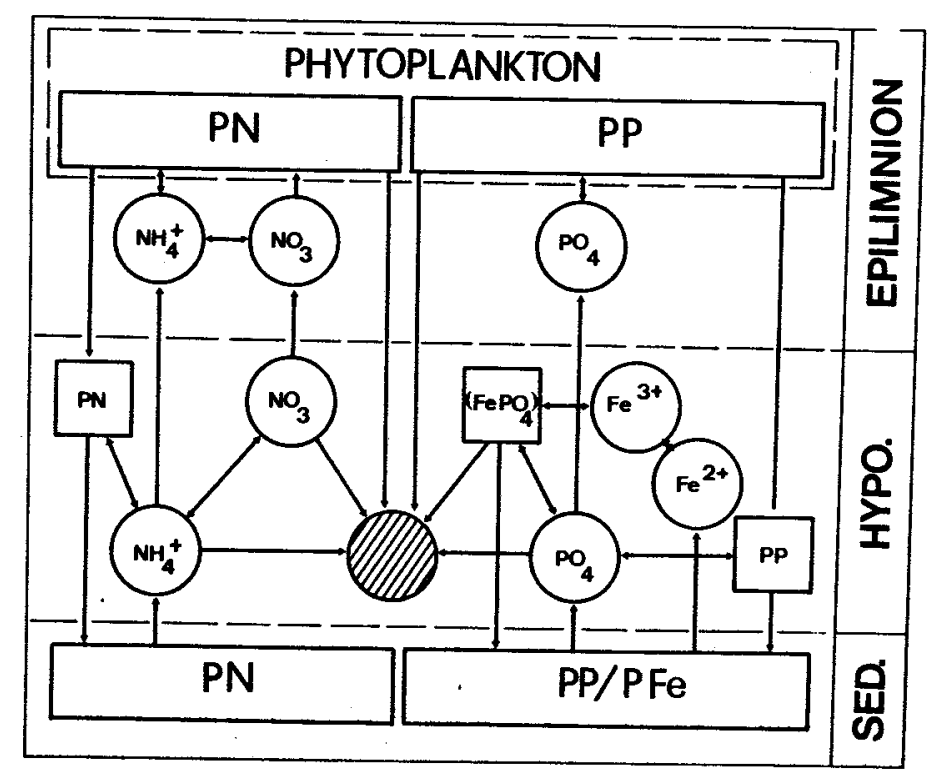

Abb. 9. Beeinflussung des Stickstoff- und Phosphorkreislaufs durch die Tiefenwasserableitung. Fig. 9. Diagram showing the influence of the siphon (shadded area) on the nitrogen and phosphorus cycle of the lake.
Wege und beschleunigt ebenfalls die $\mathrm{NH}_{4}$-Freisetzung aus den Sedimenten. Der erhöhte Export von $\mathrm{NH}_{4}, \mathrm{NO}_{3}$ und $\mathrm{PO}_{4}$ via die TWA bewirkt eine kleinere Rückdiffusion von anorganischen Nährstoffen ins Epilimnion, was zu einer verringerten Primärproduktion und einer kleineren Sedimentation von partikulärem Stickstoff (PN) und partikulärem Phosphor (PP) führt. Da durch die TWA auch partikuläres Material, das sonst unweigerlich in die Sedimente eingebaut würde, exportiert wird, wird der Einbau von PN und PP in die Sedimente zusätzlich etwas verringert. Während der Stagnationsperiode bewirkt die TWA also eine erhöhte Rücklösung und einen verringerten Einbau in die Sedimente, was im Endeffekt zu. einem kleineren Stickstoffgehalt der Sedimente und damit verbunden zu einer kleineren Rücklösung während der Zirkulationsperiode führt.

Es wurde gezeigt, dass dank der TWA während der Sommermonate die seeinterne Phosphorbelastung des Epilimnions kleiner wurde und dass die TWA auch für die Herabsetzung der Nitratkonzentration während der Zirkulationsperiode verantwortlich ist. Es wäre zu erwarten, dass dank dieser verringerten Nährstoffbelastung der trophogenen Zone auch die Primärproduktion niedriger wurde. Leider wurden direkte Produktionsmessungen nur in den Jahren 1971 und 1974 durchgeführt, in zwei Jahren also, in denen sich die Nährstoffversorgung des Epilimnions, wenn überhaupt, dann nur noch unwesentlich verändert hat. In Ubereinstimmung damit fielen die Produktionsraten mit $347 \mathrm{~g} / \mathrm{m}^{2} \mathrm{Jahr}$ im Jahre 1971 und mit $303 \mathrm{~g} / \mathrm{m}^{2}$ Jahr im Jahre 1974 beinahe gleich gross aus. Die Beobachtung, dass die epilimnische Sauerstoffmaxima während der Jahre 1968 und 1970 deutlich über denjenigen der Jahre 1971 und 1974 lagen (siehe Abb.3), dass die Nitratzehrung im Epilimnion und die Sauerstoffzehrung im Metalimnion alljährlich kleiner wurde, dass die Biomasse kleiner (siehe Abb. 8 ) und die Sichttiefe grösser wurde (siehe Abb.4), deuten darauf hin, dass die Primärproduktion in den Jahren 1968 bis 1970 höher war als in den Jahren 1971 bis 1974. Eine Beeinflussung dieser Grössen durch die unterschiedlichen, klimabedingten Schichtungsverhältnisse kann zwar nicht ausgeschlossen werden, es ist jedoch sehr wahrscheinlich, dass die Abnahme der Primärproduktion auf die nachgewiesenermassen durch die TWA bedingte Verringerung der Nährstoffbelastung des Epilimnions zurückzuführen ist.

\section{Schlussfolgerungen}

Aufgrund der obigen Ausführungen können die folgenden Schlussfolgerungen gezogen werden:

1. Es ist möglich, einen See durch die Ableitung von Tiefenwasser in seinem trophischen Charakter zu beeinflussen.

2. Durch die TWA wird der Kreislauf von anaerober Phosphorrücklösung und aerober Resedimentation durchbrochen. Dieser Prozess führt zu einer Phosphorverarmung der obersten Sedimentschicht, was eine rückläufige Entwicklung der Phosphorrücklösungsrate zur Folge hat, selbst dann, wenn die Sediment-WasserKontaktzone anaerob bleibt.

Ist die Sauerstoffkonzentration knapp über dem Seegrund höher als $3 \mathrm{mg} / \mathrm{l}$, so reagieren die Sedimente als Phosphorfalle; sinkt sie unter $1 \mathrm{mg} / \mathrm{l}$, so werden die Sedimente zur Phosphorquelle. 
3. In eutrophen Flachgewässern kann die seeinterne Phosphorbelastung die externe Belastung während der Sommerstagnation kurzfristig um mehr als das Hundertfache übertreffen.

Durch die TWA wurde diese seeinterne P-Belastung des Epilimnions sehr stark vermindert, obwohl der See in den Jahren 1971 und 1974 aufgrund der Temperaturverteilung weniger stabil geschichtet war als in den Jahren 1968 bis 1970.

4. Die TWA vermag die Phosphorkonzentration während der Zirkulationsperiode nicht zu beeinflussen. Eine Veränderung der externen Belastung bewirkt während der Vollzirkulation sehr rasch eine Veränderung in der Phosphorkonzentration.

5. Die TWA setzt sowohl die Stickstoffbelastung des Epilimnions als auch die Stickstoffkonzentration während der Vollzirkulation herab.

6. Flache, eutrophe Seen wirken auch ohne TWA nicht ganzjährig als Nährstoffallen. Während der Sommerstagnation kann der P-Export zeitweise grösser sein als die P-Zufuhr. Während der Zirkulationsphase kann der Stickstoffexport die Stickstoffzufuhr übertreffen.

7. Die Herabsetzung des Nährstoffflusses zur trophogenen Schicht führt zu einer verringerten Primärproduktion und damit zu einer niedrigeren Belastung des Hypolimnions mit organischem Material.

8. Die TWA führt zu einer Absenkung des Metalimnions und damit zu einer Volumenvergrösserung des sauerstoffreichen Epilimnions und zu einer Verringerung der anoxischen Sedimentoberfläche. Dieser Effekt ist um so grösser, je grösser das Verhältnis von der durch die TWA abgeflossenen Wassermenge zur Seeoberfläche ist und je kleiner das Verhältnis zwischen der mittleren und der maximalen Seetiefe ist.

\section{Zusammenfassung}

Es war das Ziel dieser Arbeit, am Beispiel des Mauensees zu untersuchen, wieweit sich der trophische Charakter eines Sees durch die Ableitung von Tiefenwasser beeinflussen lasse. $\mathrm{Zu}$ diesem $\mathrm{Zweck}$ wurde der See seit Inbetriebnahme der Leitung in etwa monatlichen Abständen während 5 Jahren untersucht.

Während dieser Beobachtungsperiode hat sich der Trophiegrad des Sees deutlich verändert. Sowohl die Sauerstoffübersättigung im Epilimnion als auch die Sauerstoffzehrung in den tieferliegenden Wasserschichten wurde kleiner. Die Zunahme der Transparenz bewirkte ein Absinken der Kompensationstiefe. Die vertikalen Primärproduktionsprofile wurden ausgeglichener, die epilimnische Nitratkonzentration und die Nitratzehrung zu Beginn der Sommerstagnation und die Gesamtphosphorkonzentration während der Sommerstagnation wurden deutlich kleiner.

Während der Zirkulationsperiode blieb die Phosphorkonzentration so lange unverändert, bis zusätzlich zur Ableitung des Tiefenwassers auch die externe Belastung verringert wurde. Im Gegensatz dazu ist die Gesamtstickstoffkonzentration auch bei unveränderter externer Belastung während der Zirkulationsperiode kleiner geworden. Das teilweise unterschiedliche Verhalten von Phosphor und Stickstoff wird damit erklärt, dass aus dem Sediment rückgelöster Phosphor unter aeroben Bedingungen schwerlösliche Verbindungen eingeht und daher abiotisch wieder ausgefällt wird. Der Prozess der Auteutrophierung wird beschrieben. Es wird gezeigt, dass in flachen, eutrophen Seen während des Sommers die seeinterne sedimentbürtige Phosphorbelastung der trophogenen Schicht die externe Belastung vorübergehend mehrere hundertmal übertreffen kann. Solche Seen reagieren dann vorübergehend nicht mehr als Phosphorfalle, sondern sie werden auch ohne TWA zur Phosphorquelle. In etwas geringerem Ausmass gilt während der Wintermonate das gleiche auch für den Stickstoff.

Dank dem durch die TWA künstlich sehr stark erhöhten Nährstoffexport verarmten die Sedimente an Phosphor und Stickstoff, was sich in einer deutlichen Abnahme der Rücklösungsraten auswirkte.

Die Herabsetzung des Nährstoffflusses zur trophogenen Zone führte zu einer verringerten Primärproduktion und damit zu einer niedrigeren Belastung des Hypolimnions mit organischer Substanz. Gleichzeitig wurde das Sauerstoffdefizit in der tropholytischen Zone auch deshalb kleiner, weil durch die TWA die Aufenthaltszeit des Wassers in dieser Zone von rund 180 Tagen auf nur 76 Tage verringert wurde.

\section{SUMMARY}

Mauensee is a relatively small and shallow lake located in the central part of Switzerland (see Fig. 1). Mean depth, maximum depth and lake surface area are $3.9 \mathrm{~m}, 6.8 \mathrm{~m}$ and $0.51 \mathrm{~km}^{2}$, respectively. Its drainage basin covers an area of $4.3 \mathrm{~km}^{2}$. The mean residence time of the water and the lake's Is drat $0.7 \mathrm{~g} \mathrm{P} / \mathrm{m}^{2}$ year and $19 \mathrm{~g} \mathrm{~N} / \mathrm{m}^{2}$ year, external nu bas low transparency and anoxic conditions (1) in the deeper lake strata during summer stagnation periode were characteristic for this very eutrophic
lake.

With reference to Olszewsxi [13, 14], Eschmann [4] and Pechlaner [15] it was decided to restore this lake by an artificially increased nutrient export using a hypolimnion siphon, with a flow rate of $4 \mathrm{~m}^{3} / \mathrm{min}$ (see Fig. 2). This experiment began in April 1968.

The goal of this project was to investigate the extent to which the trophic state of the lake could be influenced by this measure alone. Figure 3 clearly indicates that compared to 1968 oxygen conced the stara deeper conce $4 \mathrm{~m}$. Traspared in than $4 \mathrm{~m}$. Trasparey in during summertime (see Fig.5). The decreasing amount of lotal nitrogen (Kjeldahl nitrogen $\mathrm{NO}_{3}+\mathrm{NO}_{2}$ ) in fall and winter (see Fig.6) also suggest a positive effect of the hypolimnion drainage. Compared to results from Zimmermann photosynthesis did not decrease significantly (see Fig.7) but it is believed that in 1967 the depth of maximum photosynthesis was not located at the lake surface as reported by Zimmermann but located somewhere below the surface and above $1 \mathrm{~m}$ depth. We therefore believe that areal primary production rates were underestimated in 1967. Figure 7 also shows, that compared to 1967 the trophogenic layer expanded in 1971 and 1974.

The maximum bamass of the blue green Oscillatoria rubescens decreased from $152 \mathrm{~g} / \mathrm{m}^{2}$ in 1967 The $\mathrm{m}^{2}$ in 1974. The high total phytoplankton biomass in Augus/September 1974 (see Fig. 8) was due to $41 \mathrm{~g} / \mathrm{m}^{2}$ in 1974 . The high total phytoplankton biomass in Augus
to some very voluminous dinoflagellates (Ceratium hirundinella).

The main effects of the hypolimnion drainage were

- erosion of the hypolimnion,

- increase of the nutrient export out of the lake.

A water mass balance (Table 2) shows that due to siphoning, a small but continuous downwards flow leads to the fact that a defined volume of water which has passed the 4-m horizont leaves the lake after a residence time of only 76 days. Oxygen conditions in the hypolimnion thus improved because the remmer stagation period lasted about half a year and because the oxygen concentration because the a the intensity of the oxygen consuming processes as well as on the water's residence time in the tropholytic layer. In addition, the increased biological supply 
of oxygen to the upper part of the hypolimnion (due to the increased extention of the trophogenic layer) as well as the likely lower loading with autochthonously produced degradable organic substances might have contributed to the increased oxygen concentration in the hypolimnion.

In order to study the influence of the siphon on the nutrient content of the lake, a phosphorus mass balance has been estimated. As Table 3 indicates, the lake reacts as a phosphorus sink from October to May. During the rest of the year the phosphorus export clearly exceeds the phosphorus loading to the lake. Furthermore it is shown that during the latter period the sediments become very important phosphorus source. The phosphorus supply from the sediments exceeded by up to very important phosphorus source. The phosphorus supply from the sediments exceeded by up to
275 times the external loading in June/July 1968, in June/July 197015 times, 19716 times and in August 19744 times. The sediments reacted mostly as a phosphorus source if the oxygen concentration above the sediment surface was below $1 \mathrm{mg} \mathrm{O} / 1$, but as a phosphorus sink if it exceeded $3 \mathrm{mg} \mathrm{O} / 1$.

In summer 1968, during a periode of 28 days, the sediments released about $4560 \mathrm{~kg}$ phosphorus, 3270 of which became resedimentated or was readsorbed at the sediment surface in the following 41 days. Since the yearly external loading is only about $360 \mathrm{~kg}$, this alternation of anoxic solution and arebic fixation at the sed sediment layer. This 'snowball

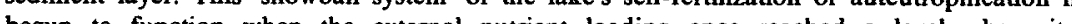
begun to function when the external nutrient loading once reached a level where it favoured transiently anoxic conditions at the sediment-water interface. That also suggests that in a eutrophic lake, the phosphorus profile in the sediments cannot serve as a simple direct memory record of the external phosphorus loading.

Since siphoning began the phosphorus expont from the lake exceeded the external supply to the lake by about $3700 \mathrm{~kg}$. This export excess caused a reduction in phosphorus at the sediment surface, which explains the decreasing rate of phosphorus release during the summer stagnation period (a) dissolution at the sediment surface. During these periods $1968,1970,1971$ and 1974 back diffusion to the epilimnion exceeded the external loading 70 to $290,32,5$ and 3 times, respectively.

A nitrogen balance can only be estimated during fall and wintertime, when oxygen and nitrate concentration are high, so that biotic nitrogen fixation as well as denitrification processes are negligable. Table 5 suggests that the sediments reacted mostly as a nitrogen source during wintertime $[(R-S)>0]$ Compared to the external nitrogen loading the net flux from the sediments to the water exceeded the external loading by a factor of 4 in 1969. Since 1970 this internal nitrogen supply was generally only a fraction of the external nitrogen input to the lake.

Figure 9 indicates how the siphon (shadded area) influences the nitrogen cycle during stagnation periode. $\mathrm{NH}_{4}{ }^{+}$is released by ammonification processes from organic particulate nitrogen (PN) in the sediments, and diffuses, into the water. The siphon removes part of this $\mathrm{NH}_{4}{ }^{+}$physically. Due to the improved oxygen supply to the strata deeper than $4 \mathrm{~m}$, it also increases the $\mathrm{NH}_{4}{ }^{+}$removal by nitrification processes. This results in a steeper concentration gradient between the interstitial wate and the water above the sediments enhancing the ammonia flux rate from the sediments to the water

On the other hand, the sedimentation rate of PN became smaller because of the lower phytoplankto density and the partiel export of PN via the siphon. The artifically increased dissolution rate and the decreased sediment fixation rate during summer sagnation period resulted in a reduction of nitrogen in the top layer of sediment. This explaines the diminishing importance of the sediments as a nitrogen source during fall and winter overturn.

It has been deduced that the siphon was responsible for the decreasing phosphorus loading by the sediments during summer stagnation as well as for the lower nitrate concentration during fall and winte overturn. Higher transparency, lower phytoplankton density, the increased extension of the trophogenic layer and lower oxygen concentrations in the epilimnion during summer stagnation suggest that compared to 1967 primary production also-decreased. Oxygen conditions in the hypolimnion improved due to the water's decreased residence time and the probably lower oxygen consumption rate in the hypolimnion.

Based on these results, the following conclusions can be made:

1. It is possible to influence a shallow lake's trophic character by siphoning off part of its bottom water.

2. Due to the yearly alternation of anoxic phosphorus release and aerobic phosphorus fixation at the sediment-water interface, phosphorus becomes accumulated in the very top layer of the sediments. 3. As more and more phosphorus becomes available in the top layer of the sediment the lake's internal phosphorus cycle becomes accelerated. Thus, this accumulation process causes a selfinaintaining auteutrophication of a lake as soon as the external nutrient loading reaches such a level maintaining auteutrophication of a lake as soon as the external nutrient
that it favours transiently anoxic conditions at the sediment-water interface.

that it favours transiently anoxic conditions at the sediment-water interface. 4. In a shallow eutrophic lake the external loading by more than a hundredfold.

5. During the summer stagnation period, such a lake no longer reacts as a phosphorus sink. Even without a siphon the phosphorus export exceeds the external phosphorus supply to the lake.

6. The same is true for nitrogen during fall and winter overturn.

7. Due to the drastically increased nutrient export, siphoning causes a nutrient reduction in the top 7. Due to the drastically increased nutrient export, siphoning causes a nutrient red
sediment layer which decreases the back diffusion rate from the sediments to the water.

8. As long as the nutrient supply to the trophogenic layer is the rate limiting factor of primary production, siphoning will also necessarily decrease the primary production of a lake.

9. Lower primary production, increased biological oxygen supply to the hypolimnion and shorter residence time of the water in this layer, leads to an improvement of the oxygen conditions in the hypolimnion, when siphoning of the bottom water is practised.

Eigentlicher Initiant dieses Projektes war Dr.F.Adam, Kantonschemiker des Kantons Luzern. Eigendicher vom Eidg Amt für Umweltschutz sowie vom Kanton Luzern. Die Leitung und das Auslaufbauwerk wurden projektiert vom schutz sowie vom Kanton Luzern. Die Leitung und

Lieselotte Mühlebach-Krähenbühl, A. Mares, E. Grieder sowie den Mitarbeitern des kantonalen Laboratoriums Luzern bin ich für die Durchführung der Probenahmen und die Bereitstellung der Analysendaten, Fräulein Beatrix Egli für die Bearbeitung des Planktons zu Dank verpflichtet.

Danken möchte ich auch Frau Dr. von Schumacher für ihr stetes Interesse an unserer Arbeit und dafür dass sie uns die zur Durchführung der Probenahmen notwendigen Boote zur Verfügung dafur, dass

Fräulein Heidi Bolliger danke ich für die saubere Ausführung der graphischen Darstellungen.

Besonderer Dank gebührt Dieter Imboden und Mike Kavanaugh für die kritische Durchsicht des Manuskripts und die angebrachten Verbesserungsvorschläge.

\section{LITERATURVERZEICHNIS}

[1] АмвÜн, H., Die praktische Anwendung der elektrochemischen Sauerstoffbestimmung im Wasser, Schweiz. Z. Hydrol. 22, 23-39 (1960).

[2] АмвÜнL H., und SCHмID, M., Bestimmung geringster Mengen von Phosphation im Wasser von Binnenseen, Schweiz. Z. Hydrol. 27, 172-183 (1965).

[3] BREZONIK, P., Nitrogen: Sources and Transformations in Natural Waters, in: Nutrients in natural waters (Ed. H.A. Allen and J.R. Kramer; John Wiley \& Sons, Inc., New York a.o. 1972)

[4] Eschmann, K.H., Die Sanierung des Wilersees durch Ableitung des Tiefenwassers, Gesundheitstechnik, Zürich 3, 125-128 (1969).

[5] GÄCHTER, R., Zwischenbericht über den gegenwärtigen Zustand des Mauensees, die getroffenen Sanienungsmassnahmen und die Auswirkungen der Tiefenwasserableitung, Bericht EAWAG 4318 (1971), unveröffentlicht.
(

[6]GÄCHTER, R., und FURRER, O.J., Der Beitrag der Landwirtschaft zur Eutrophierung der Gewässer in der Schweiz, Schweiz. Z. Hydrol. 34, 41-70 (1972)

[7] GäCHTER, R., Phosphorhaushalt und planktische Primärproduktion im Vierwaldstättersee (Horwer Bucht), Schweiz. Z. Hydrol. 30, 1-66 (1968).

[8] GÄCHTER, R., Die Bestimmung der Tagesraten der planktischen Primärproduktion - Modelle und In-situ-Messungen, Schweiz. Z. Hydrol. 34, 211-244 (1972).

[9] ImBoden, D.M., Limnologische Transport- und Nährstoffmodelle, Schweiz. Z. Hydrol. 35, 29-68 (1973).

[10] Krummenacher, Th., Die Nährstoffbilanz des Alpnachersees, Dissertation ETH Zürich, in Vorbereitung.

[11] MülukR, R., und Wiedemann, O., Die Bestimmung des Nitrations im Wasser, Jb. Vom Wass. 12 , 247-27I (1955) 
[12] Oнце, W., Sulfat als "Katalysator» des limnischen Stoffkreislaufes, Vom Wass. 21, 13-32 (1954).

[13] OLszewsk1, P., Versuch einer Ableitung des hypolimnischen Wassers aus einem See. Ergebnisse des ersten Versuchsjahres, Verh. int. Ver. Limnol. 14, 855-861 (1961).

[14] OlszewsKı, P., Die Ableitung des hypolimnischen Wassers aus einem See, Föderation Europäischer Gewässerschutz, Inform.-Blatt 14, S. 87-89 (1967).

[15] Pechlaner, R., Die Restaurierung des Piburger Sees (Tirol), Carinthia, Sonderh. 31, 97-115 (1971).

[16] SснміD, M., und АмвüHL, H., Bestimmung geringster Mengen von Gesamtphosphor im Wasser von Binnenseen, Schweiz. Z. Hydrol. 27, 183-192 (1965).

[17] Schwoerbel, J., Methoden der Hydrobiologie (Verlag Kosmos, Stuttgart 1966).

[18] Stadelmann, P., Stickstoffkreislauf und Primärproduktion im mesotrophen Vierwaldstättersee (Horwer Bucht) und im eutrophen Rotsee, mit besonderer Berücksichtigung des Nitrats als limitierendem Faktor, Schweiz. Z. Hydrol. 33 (1971).

[19] Steemann-Nielsen, E., The Use of the Radioactive Carbon $\left({ }^{14} \mathrm{C}\right)$ for Measuring of Organic Production in the Sea, J. Cons. int. Explor. Mer 18, 117-140 (1952).

[20] Strickland, J.H.D., und Parsons, T.R., A Practical Handbook of Seawater Analysis, Bull. Fish. Res. Board. Can. 167, 41-44 (1968).

[21] TESSENOW, U., Lösungs-, Diffusions- und Sorptionsprozesse in der Oberflächenschicht von Sedimenten. I. Ein Langzeitexperiment unter aeroben und anaeroben Bedingungen im Fliessgleichgewicht, Arch. Hydrobiol., Suppl. 38, 353-398 (1972).

[22] WINKıER, L.W., Die Bestimmung des im Wasser gelösten Sauerstoffs und die Löslichkeit des Sauerstoffs im Wasser, Ber. dt. Chem. Ges. 21, 2845 (1888), und 22, 1764 (1889).

[23] ZimmermanN, U., Ökologische und physiologische Untersuchungen an der planktischen Blaualge Oscillatoria rubescens D.C. unter besonderer Berücksichtigung von Licht und Temperatur, Schweiz. Z. Hydrol. 31, 1-58 (1969).

Adresse des Autors:

Dr. RenéGächter,Seenforschungslaboratorium derEAWAG/ETH,CH-6047Kastanienbaum,Switzerland. 